\title{
A table-top femtosecond time-resolved soft x-ray transient absorption spectrometer
}

\author{
Zhi-Heng Loh, Munira Khalil, ${ }^{\dagger}$ Raoul E. Correa, and Stephen R. Leone*
}

\begin{abstract}
Departments of Chemistry and Physics, University of California, Berkeley, CA 94720, and Chemical Sciences Division, Lawrence Berkeley National Laboratory, 1 Cyclotron Road, Berkeley, CA 94720
\end{abstract}

\begin{abstract}
A laser-based, table-top instrument is constructed to perform femtosecond soft x-ray transient absorption spectroscopy. Ultrashort soft x-ray pulses produced via high-order harmonic generation of the amplified output of a femtosecond Ti:sapphire laser system are used to probe atomic core-level transient absorptions in atoms and molecules. The results provide chemically specific, time-resolved dynamics with sub-50-fs time resolution. In this setup, high-order harmonics generated in a Ne-filled capillary waveguide are refocused by a gold-coated toroidal mirror into the sample gas cell, where the soft x-ray light intersects with an optical pump pulse. The transmitted high-order harmonics are spectrally dispersed with a home-built soft x-ray spectrometer, which consists of a gold-coated toroidal mirror, a uniform-line spaced plane grating, and a soft x-ray CCD camera. The optical layout of the instrument, design of the soft x-ray spectrometer, and spatial and temporal characterization of the high-order harmonics are described. Examples of static and time-resolved photoabsorption spectra collected on this apparatus are presented.
\end{abstract}

\footnotetext{
$\dagger$ Present address: Department of Chemistry, University of Washington, Box 351700, Seattle, WA 98195

* Corresponding author. Electronic mail: srl@berkeley.edu
} 


\section{INTRODUCTION}

Recent advances in ultrashort-pulsed x-ray source development have enabled the study of ultrafast phenomena in the $\mathrm{x}$-ray domain. ${ }^{1-4}$ Ultrafast $\mathrm{x}$-ray absorption measurements performed at third-generation synchrotron light sources elucidate both atomic displacements and changes in electronic structure accompanying photoexcitation of solvated molecules ${ }^{5,6}$ and solid state materials. ${ }^{7}$ By employing a pulse-slicing scheme, ${ }^{8}$ synchrotron pulses of sub-picosecond temporal duration can be applied to ultrafast x-ray absorption $^{9}$ and diffraction ${ }^{10}$ experiments. Femtosecond x-ray pulses produced at freeelectron laser (FEL)-based facilities enable the direct visualization of atomic motion in ultrafast photoinduced phase transitions. ${ }^{11,12}$ In addition, the intense $\mathrm{x}$-ray pulses produced at FELs ${ }^{13}$ also hold great promise for x-ray-initiated pump-probe experiments.

In the realm of table-top experiments, femtosecond hard $\mathrm{x}$-ray pulses from laserproduced plasmas are used to investigate the gas-phase photodissociation dynamics of small molecules, ${ }^{14}$ as well as to observe the structural rearrangement in solids ${ }^{15-17}$ and to probe solvated molecules ${ }^{18}$ upon laser excitation. Ultrashort soft x-ray pulses produced by high-order harmonic generation ${ }^{19,20}$ (HHG) are used as single-photon ionization probes of molecular dynamics in the gas phase ${ }^{21,22}$ and on surfaces, ${ }^{23-25}$ thereby circumventing multiple resonant interactions that occur with multiphoton ionization probing. The use of few-cycle near-infrared pulses for HHG have led to the generation of isolated attosecond pulses, ${ }^{26}$ which allow the investigation of electron dynamics with unprecedented time resolution. ${ }^{27-29}$

While the set of discrete peaks in HHG-produced soft x-ray radiation presents an attractive application in kinetic energy-resolved photoelectron spectroscopy, a spectral 
continuum required for absorption spectroscopy has to-date been discussed primarily in the HHG cut-off region with few-cycle driver pulses. Hence it is not surprising that spectroscopy with high-order harmonics has been largely limited to photoelectron probing, and photoabsorption spectroscopy has not been demonstrated until recently. ${ }^{30-35}$

In core-level photoabsorption spectroscopy, an incident x-ray photon promotes an electron residing in a core level to an unoccupied valence orbital. In a spectroscopic picture, the $\mathrm{x}$-ray photon promotes the atom/molecule from an initial state with completely-filled core levels to a core hole-excited final state. (Note that the initial state does not necessarily have to be the electronic ground state, since valence excitations can also exist.) Core-level photoabsorption spectroscopy therefore combines the elemental specificity of core-level probing with the sensitivity to changes in the valence electronic structure, i.e. chemical environment, of the atom/molecule induced by photophysical or photochemical transformations. The resonant transitions measured in transient absorption spectroscopy make the application of ultrashort broadband probe pulses compatible with this technique. On the other hand, the bound-free transitions measured in photoelectron spectroscopy limit the spectral bandwidth that can be used in the probe pulse and still achieve adequate spectral resolution. Moreover, spectral dispersion in transient absorption spectroscopy can be performed after the sample, thereby circumventing the need for a time-delay-compensated monochromator, ${ }^{36,37}$ which is used to select a single harmonic for photoelectron spectroscopy.

An early application of high-order harmonics to absorption spectroscopy involves the collection of static absorption spectra at the $M_{2 / 3}$-edge of first-row transition metal thin films. ${ }^{30}$ The spectra are obtained by recording the changes in the transmission at the peak 
of the individual harmonics when the sample is introduced. More recently, the application of high-order harmonics with photon energies up to $3.5 \mathrm{keV}$ allowed interatomic distances in metal and semi-metal thin foils to be retrieved ${ }^{31}$ from their static extended $\mathrm{x}$ ray absorption fine structure (EXAFS) spectra. $^{38}$ In the realm of time-resolved measurements, femtosecond soft x-ray transient absorption spectroscopy using high-order harmonics was recently demonstrated and applied by our group to investigate laseratom/molecule interactions in the nonperturbative, strong-field regime. ${ }^{32-34}$ In a separate effort by Spielmann and co-workers, real-time atomic displacements following optical excitation of a $\mathrm{Si}$ thin foil were observed by femtosecond time-resolved EXAFS spectroscopy employing high-order harmonics. ${ }^{35}$ While such experiments have only been performed with soft $\mathrm{x}$-rays in the range of $50 \mathrm{eV}-100 \mathrm{eV}$ to date, these studies demonstrate the applicability of high-order harmonics and their complementarity to other pulsed $\mathrm{x}$-ray sources for use as a probe in ultrafast $\mathrm{x}$-ray absorption spectroscopy.

Here we present a detailed description of a table-top, laser-based instrument for highorder harmonic transient absorption spectroscopy. The remainder of the paper is organized as follows. The laser system, optical layout of the experiment, and the HHG setup are described in Sections IIA, IIB, and IIC, respectively. The vacuum apparatus, design of the home-built soft x-ray spectrometer and the data acquisition procedures are given in Sections IID, IIE, and IIF, respectively. The high-order harmonic spectra are shown in Section IIIA, followed by a discussion of the wavelength-tunability of highorder harmonics in Section IIIB. Results obtained from photon flux and spatial beam profile measurements are given in Sections IIIC and IIID, respectively. Examples of nonresonant and resonant static photoabsorption spectra collected on the apparatus are 
presented in Section IIIE. The transient absorption spectrum of $\mathrm{Xe}^{2+}$ produced by optical strong-field ionization of Xe atoms is presented in Section IIIF, along with results from the temporal characterization of the high-order harmonics via pump-probe crosscorrelation.

\section{EXPERIMENTAL SETUP}

An overview of the experimental apparatus is illustrated in Fig. 1. The main components are an amplified Ti:sapphire femtosecond laser system, a capillary waveguide in which the high-order harmonics are produced, an interaction region where the optical pump and soft x-ray probe beams intersect at the sample, and a home-built soft x-ray spectrometer. A detailed description of each of these components, along with the data acquisition procedures, will be provided in this section.

\section{A. FEMTOSECOND LASER SYSTEM AND PULSE DIAGNOSTICS}

Femtosecond pulses employed in the experiment are produced by a commercial amplified Ti:sapphire laser system. The average output power is $2.6 \mathrm{~W}$ at $1 \mathrm{kHz}$ repetition rate, the spectral bandwidth is $31 \mathrm{~nm}$ FWHM with a center wavelength of 800 $\mathrm{nm}$, and the pulse duration is $<45 \mathrm{fs}(\mathrm{FWHM})$. The beam waist $w_{0}$ is measured using the scanning knife-edge method and is found to be $5.1 \mathrm{~mm}$. The temporal profile of the amplified pulse is characterized using either single-shot autocorrelation or spectral-phase interferometry for direct electric field reconstruction (SPIDER). ${ }^{39}$ The former is used as a daily diagnostic for the amplifier, whereas the latter is used when knowledge of the accurate temporal profile is critical, for instance, when characterizing the pump pulse 
used in strong-field ionization experiments. In addition, the intensity stability of the amplifier output is monitored by sending the beam through a $200 \mu \mathrm{m}$ thick Type I BBO crystal $(\theta=29.2 \mathrm{deg})$ and measuring the power of the frequency-doubled output on a power meter. Over the course of one hour and with a sampling interval of $1 \mathrm{~s}$, the root mean squared (RMS) fluctuation is typically $<0.2 \%$ with a typical average power of $>0.8$ W for the frequency-doubled light. The intensity stability of the amplifier output is also monitored on a pulse-to-pulse basis by sending the $800 \mathrm{~nm}$ leakage through the first turning mirror after the amplifier into another BBO crystal and detecting the second harmonic output with a fast photodiode. Over a period of $1 \mathrm{~s}$, the pulse-to-pulse RMS fluctuation is typically $<2 \%$.

\section{B. OPTICAL LAYOUT}

The output from the Ti:sapphire laser system is sent to a 20:80 beamsplitter (Newport, 20RQ00UB.2) to produce the optical pump and HHG driver beams, respectively. The relative timing between the optical pump and HHG driver pulses is varied by means of a computer-controlled motorized translation stage (Aerotech, ALS130) positioned in the pump beam path. A zero-order half-waveplate (CVI, QWPO-800$08-2-\mathrm{R} 10$ ) in the path of the pump beam is used to vary the relative polarization between the pump and probe beams. In addition, an iris diaphragm can be used to control the energy of the $800 \mathrm{~nm}$ light used for excitation. In strong-field ionization experiments, a UV fused silica lens with a $45 \mathrm{~cm}$ focal length (CVI, PLCX-25.4-206.0-UV-800) is used to focus the $800 \mathrm{~nm}$ pump beam before it enters the sample chamber. The input window consists of a $1 \mathrm{~mm}$ thick UV fused silica substrate with broadband anti-reflection coating 
for $670 \mathrm{~nm}-1064 \mathrm{~nm}$ on both optical surfaces (CVI, W2-PW-0706-UV-670-1064-0). A pick-off mirror (Newport, 10B20UF.25) positioned $10 \mathrm{~cm}$ before the interaction region allows the optical pump beam to intersect with the soft $\mathrm{x}$-ray probe beam at the sample with a $2^{\circ}$ crossing angle (Fig. 2). The focal spot size at the interaction region is determined via relay imaging to a CCD beam profiler; typical beam waists along the horizontal and vertical directions are $30 \mu \mathrm{m}$ and $32 \mu \mathrm{m}$, respectively. The confocal parameters of the $800 \mathrm{~nm}$ pump beam in the horizontal and vertical directions are measured to be $6.4 \mathrm{~cm}$ and $6.8 \mathrm{~cm}$, respectively.

The $800 \mathrm{~nm}$ beam to drive HHG is focused by a $68 \mathrm{~cm}$ focal length UV fused silica lens (CVI, PLCX-25.4-309.1-UV-800) into the capillary waveguide in which the highorder harmonics are produced. A pair of $0.2 \mu \mathrm{m}$ thick Al foils (Lebow Company) mounted on a push-pull and positioned $60 \mathrm{~cm}$ after the capillary exit is used to remove the residual HHG driver pulse that co-propagates with the soft x-ray light from the exit of the capillary waveguide. Alternatively, a broadband $800 \mathrm{~nm}$ high reflector mounted on a push-pull (Newport, 10B20UF.25) can be used to direct the residual $800 \mathrm{~nm}$ beam from the capillary output through an output window towards a beam profiler (Spiricon, LBWFW-SCOR20), thereby enabling the spatial mode of the coupled HHG beam to be monitored and optimized while the entire apparatus is under vacuum. A spectrometer (Ocean Optics, HR2000CG-UV-NIR) can be positioned in place of the beam profiler to measure the spectrum of the HHG driver pulse emerging from the capillary waveguide.

The high-order harmonics, which diverge as they emerge from the exit of the capillary waveguide, are transmitted by the $\mathrm{Al}$ foils and refocused by a gold-coated toroidal mirror (Jobin Yvon) into the sample gas cell. The toroidal mirror is mounted on a 
motorized kinematic mount to enable its alignment while the apparatus is under vacuum. The mirror is used at an incidence angle of $85^{\circ}$ and has a clear aperture of $7 \mathrm{~cm}(\mathrm{~h}) \times 1$ $\mathrm{cm}(\mathrm{v})$. Its reflectivity is expected to vary between $81 \%$ and $85 \%$ over a photon energy range of $30 \mathrm{eV}-100 \mathrm{eV} .{ }^{40}$ The radii of curvature are $749.910 \mathrm{~cm}(\mathrm{~h})$ and $5.7138 \mathrm{~cm}(\mathrm{v})$, giving effective source-to-mirror and mirror-to-image distances of $72 \mathrm{~cm}$ and $60 \mathrm{~cm}$, respectively, in addition to a displacement of $0.4 \mathrm{~cm}$ between the horizontal and vertical foci at the image. Ray tracing yields estimated confocal parameters of $2.0 \mathrm{~cm}$ and $2.2 \mathrm{~cm}$ for the horizontal and vertical axes, respectively. These values are significantly larger than the foci displacement; as such, the displacement of the horizontal and vertical foci is not expected to have a significant effect on the performance of the instrument.

The sample gas cell is mounted on a motorized $x y z$ translation stage, which allows the beam entrance and exit pinholes of the gas cell to be aligned to the optical pump and soft x-ray probe beams under vacuum. With the Al foils moved out of the HHG beam path so that the residual $800 \mathrm{~nm}$ light is incident on the sample gas cell, spatial and temporal overlap of the pump and probe beams can be found by translating, into the beam path, a $100 \mu \mathrm{m}$ thick Type I BBO crystal $(\theta=29.2 \mathrm{deg})$ positioned beside the sample gas cell. The motorized linear stage along this translation axis is equipped with linear encoding (Physik Instrumente, M-665.2PM), which enables the sample gas cell to be moved into and out of the beams with high bidirectional repeatability. To prevent laser-induced damage to the crystal by the intense $800 \mathrm{~nm}$ beams while finding temporal overlap, an OD2 reflective neutral density filter is inserted into the beam path before the 20:80 beamsplitter. The spatial and temporal overlap of the pump and probe beams can be further optimized by monitoring the absorbance of the $\mathrm{Xe}^{+}$ion at $55.4 \mathrm{eV}$. On a daily 
basis, the temporal overlap between the two pulses varies by $<5$ fs. After transmitting through the sample, the high-order harmonics are detected by a home-built soft $\mathrm{x}$-ray spectrometer. A $0.2 \mu \mathrm{m}$ thick $\mathrm{Al}$ foil is mounted at the entrance of the spectrometer to prevent the $800 \mathrm{~nm}$ pump beam from entering the spectrometer.

\section{HIGH-ORDER HARMONIC GENERATION}

High-order harmonics in the soft $\mathrm{x}$-ray region are generated by focusing $80 \%$ of the output from the Ti:sapphire amplifier into a UV fused silica capillary waveguide (Wilmad-LabGlass) filled with argon or neon. Capillary waveguides allow phasematched HHG over an extended interaction length, leading to an enhanced photon flux and excellent spatial mode quality for the output beam. ${ }^{41,42}$ The capillaries used in experiments have an internal diameter of $150 \mu \mathrm{m}$ and a length of $8 \mathrm{~cm}$. The capillary inner diameter is significantly larger than the $800 \mathrm{~nm}$ wavelength of the HHG driver light, which implies that waveguiding occurs by multiple grazing incidence reflections

against the inner wall of the capillary. ${ }^{43}$ Careful mode-matching is necessary to ensure optimal coupling of the $\mathrm{HHG}$ beam into the lowest $\mathrm{EH}_{11}$ mode of the capillary waveguide; for an evacuated capillary, the HHG beam needs to be focused to a beam waist of $w_{0}=0.6435 a$, where $a$ is the internal radius of the capillary. ${ }^{44}$ In our case, it is found that a UV fused silica lens with a focal length of $68 \mathrm{~cm}$ allows focusing of the 800 $\mathrm{nm}$ HHG driver beam to a beam waist of $47 \mu \mathrm{m}$, which is very close to the optimal $w_{0}$ value of $48 \mu \mathrm{m}$. The capillary waveguide has a typical throughput at $800 \mathrm{~nm}$ of $70 \%$ under vacuum; when filled with gas, the typical throughput reduces to $40-60 \%$. Laserdrilled holes located in the sidewalls at $5 \mathrm{~mm}$ from each end of the capillary serves as the 
gas inlets. ${ }^{45}$ The $5 \mathrm{~mm}$ segments on each end of the capillary enables a high gas pressure to be maintained in the central segment, while keeping a relatively low gas density at the front and rear ends of the capillary. A Baratron pressure transducer (MKS, Micro 890) located $\sim 0.5 \mathrm{~m}$ upstream of the capillary is used to measure the inlet pressure of the noble gas used for HHG.

The amount of energy used for HHG can be varied by means of an iris diaphragm positioned $43 \mathrm{~cm}$ before the entrance of the capillary waveguide. Varying the amount of input energy and therefore the plasma density in the capillary waveguide allows the photon energy of the HHG output to be tuned (see Section IIIB). Moreover, it is found that irising down the HHG driver beam often leads to a two- to five-fold increase in the photon flux at the peak of a harmonic. This enhancement could originate from spatial filtering of the input beam that results in better coupling into the capillary waveguide. ${ }^{46}$ For $\mathrm{Ar}$ and $\mathrm{Ne}$, the optimal input energies vary from $0.8 \mathrm{~mJ}-1.4 \mathrm{~mJ}$ and $1.5 \mathrm{~mJ}-1.8$ $\mathrm{mJ}$, respectively. The intensities of the HHG driver pulse therefore range from $5 \times 10^{14}$ $\mathrm{W} / \mathrm{cm}^{2}$ to $1 \times 10^{15} \mathrm{~W} / \mathrm{cm}^{2}$.

The wavelength-integrated soft x-ray photon flux is measured using a calibrated soft X-ray photodiode (IRD, AXUV100AL3). Alternatively, the photon flux of an individual harmonic can be estimated from the total number of counts detected within a certain energy bandwidth on the spectrometer CCD camera. Scanning knife edge measurements are used to measure the beam waist of the individual harmonics at the sample focus. Finally, the wavelength-integrated far-field spatial mode of the soft x-ray beam can be directly visualized by positioning a soft x-ray CCD camera $30 \mathrm{~cm}$ after the sample focus. 


\section{VACUUM APPARATUS AND SAMPLE GAS CELL}

The experimental apparatus comprises three vacuum chambers (Fig. 1). The first chamber is pumped by a $550 \mathrm{~L} / \mathrm{s}$ turbomolecular pump (Varian, V550) and houses the toroidal mirror used to refocus the soft x-ray light into the interaction region. The same turbomolecular pump evacuates the rear end of the capillary waveguide. The chamber pressure remains below $1 \times 10^{-4}$ Torr $\left(1.3 \times 10^{-2} \mathrm{~Pa}\right)$ during HHG. The second chamber is pumped by a $1000 \mathrm{~L} / \mathrm{s}$ turbomolecular pump (Varian, V1001) and contains the sample gas cell. Finally, the spectrometer chamber is pumped by a $70 \mathrm{~L} / \mathrm{s}$ turbomolecular pump (Varian, V70). The $550 \mathrm{~L} / \mathrm{s}$ and $70 \mathrm{~L} / \mathrm{s}$ turbomolecular pumps are backed by a common scroll pump (Varian, TriScroll300). The same scroll pump also evacuates the front end of the capillary waveguide. The $1000 \mathrm{~L} / \mathrm{s}$ turbomolecular pump is backed by its own scroll pump (Varian, TriScroll300). Each of the chambers is equipped with an inverted magnetron pressure gauge (Varian, IMG-100). The base pressures of the toroidal mirror, sample, and spectrometer chambers are $2 \times 10^{-8}$ Torr $\left(2.7 \times 10^{-6} \mathrm{~Pa}\right), 7 \times 10^{-9}$ Torr $(9.3 \times$ $\left.10^{-7} \mathrm{~Pa}\right)$, and $1 \times 10^{-8}$ Torr $\left(1.3 \times 10^{-6} \mathrm{~Pa}\right)$, respectively.

The cylindrically shaped gas cell, machined from polytetrafluoroethylene (PTFE), has an internal diameter of $2 \mathrm{~mm}$ and its axis is aligned perpendicularly to the beam propagation axis (Fig. 3). The nominal sample path length of the gas cell is therefore 2 $\mathrm{mm}$. The vanishing transmission of soft $\mathrm{x}$-ray light with photon energies in the range of $10 \mathrm{eV}-100 \mathrm{eV}$ through optically transparent materials prevents the use of entrance and exit windows for the sample gas cell. Instead, beam entrance and exit pinholes as small as $150 \mu \mathrm{m}$ in diameter (typically $200 \mu \mathrm{m}$ diameter) are drilled into the wall of the gas cell to allow the pump and probe beams to enter and exit the cell. With a wall thickness of 1 
$\mathrm{mm}$, the gas cell allows a sample pressure as high as 100 Torr to be achieved while the chamber pressure remains below $1 \times 10^{-3}$ Torr $(0.13 \mathrm{~Pa})$. For example, for a He sample pressure of 100 Torr and beam entrance and exit pinholes of $150 \mu \mathrm{m}$ diameter, conductance calculations give an estimated gas pressure of $8.3 \times 10^{-4}$ Torr $(0.11 \mathrm{~Pa})$ in the immediate vicinity of the gas cell. From the dimensions of the sample chamber, the estimated contribution from the gas density outside the gas cell to the total absorption signal is $<1 \%$ and can therefore be neglected. While the absorption due to the gas density outside the flowing gas cell is negligible, however, the pressure gradient present along the beam propagation axis in such a (quasi-static) flowing gas cell needs to be taken into account. Consideration of this pressure gradient results in a modification of the exponent in the Beer-Lambert law, such that the transmission $T$ of the soft x-ray probe through the sample gas cell is now given by $T=e^{-\sigma \int n(z) d z}=e^{-n_{\text {eff }} \sigma L}$, where $n(z)$ is a function representing the sample gas density along the beam propagation axis, $n_{\text {eff }}$ is the effective sample gas density, and $L$ is the nominal sample path length. The sample gas densities

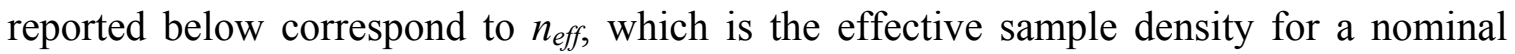
sample path length of $L$. It is important to note that the above modification to the BeerLambert law has no effect on the measured absorption cross-section; the absorption cross-sections obtained from this instrument can be compared directly to values reported in the literature.

Finally, a stream of Ar gas incident on the pick-off mirror used to overlap the pump and probe beams at the interaction region is introduced via a $100 \mu \mathrm{m}$ diameter nozzle positioned $5 \mathrm{~mm}$ before the optic; this arrangement prevents condensation and subsequent laser-induced decomposition of the molecular sample on the turning mirror, 
since peak intensities as high as $5 \times 10^{11} \mathrm{~W} / \mathrm{cm}^{2}$ at the optic can be reached in the case of strong-field ionization experiments with $800 \mathrm{~nm}$ light.

\section{E. SOFT X-RAY SPECTROMETER}

The home-built soft $\mathrm{x}$-ray spectrometer is designed to achieve a spectral resolution of $0.2 \mathrm{eV}$ FWHM in the photon energy range of $25 \mathrm{eV}-70 \mathrm{eV}$, giving a resolving power of $E / \Delta E=300$ at $60 \mathrm{eV}$. A higher spectral resolution can be achieved, for instance, by increasing the dispersion power of the grating or by increasing the distance between the grating and the $\mathrm{CCD}$ detector plane. For the purposes of investigating ultrafast molecular dynamics, however, a spectral resolution of $0.2 \mathrm{eV}$ is adequate.

The home-built soft x-ray spectrometer consists of a gold-coated toroidal mirror, a uniform-line spaced grating, and a soft x-ray CCD camera. Note that the focal point in the sample cell serves as the entrance slit to the spectrometer, and that the spectrometer resolution is therefore partially determined by the focal spot size of the soft x-ray beam at the sample position. The gold-coated toroidal mirror (Jobin Yvon) is positioned $32 \mathrm{~cm}$ after the sample focus and is used to refocus the soft x-ray light transmitted by the sample onto the CCD camera, which is positioned $30 \mathrm{~cm}$ after the toroidal mirror. The toroidal mirror is mounted at an incidence angle of $85^{\circ}$ and has a clear aperture of $1 \mathrm{~cm}(\mathrm{~h}) \times 4 \mathrm{~cm}$ (v). Its radii of curvature are $239.710 \mathrm{~cm}(\mathrm{~h})$ and $2.6580 \mathrm{~cm}(\mathrm{v})$.

At the expense of not achieving a flat-field focus at the detector, a uniform-line spaced gold-coated grating with a groove density of 600 lines $/ \mathrm{mm}$ and a blaze angle of $2.6^{\circ}$ (Zolo Tech) is used to spectrally disperse the soft x-ray light onto the CCD camera. The grating, positioned $10 \mathrm{~cm}$ after the toroidal mirror, is mounted on a motorized 
rotation stage (Physik Instrumente, M035.D01), which allows the optimal spectral resolution to be achieved by adjusting the angle of incidence. Ray tracing suggests that the incidence angle needs to be varied from $83.5^{\circ}-85.2^{\circ}$ for a photon energy range of 25 $\mathrm{eV}-70 \mathrm{eV}$. The foci for the various photon energies are shown in Fig. 4; the straight line drawn through the foci represents the plane of the CCD array. The deviation of the foci from the plane of the CCD array for the various photon energies is shown in the inset of Fig. 4. Even though a flat-field focus is not achieved, the maximum deviation of the foci from the plane of the CCD array remains less than $230 \mu \mathrm{m}$. This deviation is negligible ( $\sim 0.1 \%)$ compared to the grating-to-CCD plane distance; as such, the degradation in spectral resolution is expected to be insignificant $(<1 \%)$.

The spectrally dispersed soft x-ray light is detected by a back-illuminated soft x-ray CCD camera (Princeton Instruments, PI-SX). The CCD array is thermoelectrically cooled to $-60{ }^{\circ} \mathrm{C}$ and consists of $1340 \times 100$ pixels, with an individual pixel size of $20 \mu \mathrm{m} \times 20$ $\mu \mathrm{m}$. A pair of $0.2 \mu \mathrm{m}$ thick Al foils of dimensions $30 \mathrm{~mm} \times 3 \mathrm{~mm}$ is placed directly in front of the mounting plate of the $\mathrm{CCD}$ array to minimize stray light incident on the detector and to prevent condensation of the molecular sample onto the cooled CCD array. Because the toroidal mirror refocuses the soft x-ray light onto the CCD array, all the light can be concentrated onto less than five pixel rows in the sagittal plane (this is the plane orthogonal to the spectral dispersion plane), yielding a large count rate over a relatively small region of the $\mathrm{CCD}$ array. Over a photon energy range of $30 \mathrm{eV}-100 \mathrm{eV}$, the quantum efficiency of the $\mathrm{CCD}$ camera according to the manufacturer specification remains approximately constant at $\sim 40 \%$. Note that knowledge of the absolute quantum 
efficiency as a function of photon energy is not necessary in our case, since the quantity of interest is the transmission and not the absolute photon flux.

Wavelength calibration of the spectrometer is performed by locating the pixel positions of the $\mathrm{Al}$ foil high-energy cut-off at $72.64 \mathrm{eV}$ (Al $L_{3}$-edge) in the first four diffraction orders ${ }^{47}$ and the $\mathrm{Xe}^{+2} P_{3 / 2} \rightarrow{ }^{2} D_{5 / 2}$ peak at $55.4 \mathrm{eV}$ in the first two diffraction orders ${ }^{48}$ and employing a fitting function based on the diffraction grating equation. This procedure yields a calibration error of $\leq 0.05 \mathrm{eV}$. A typical calibration curve is shown in Fig. 5.

Finally, it is important to note that the spectrometer directly measures the transmission as a function of photon energy, and any measured absorption lineshape therefore corresponds to a convolution of the transmission function with the Gaussian spectrometer resolution function. Hence the measured optical density (absorbance) is given by

$$
\mathrm{OD}_{\text {meas }}(E)=-\log \left[\operatorname{Exp}\left(-n_{e f f} \sigma(E) L\right) * \sqrt{\frac{4 \ln 2}{\pi \Delta^{2}}} \operatorname{Exp}\left(-\frac{4 \ln 2 E^{2}}{\Delta^{2}}\right)\right],
$$

where $n_{\text {eff }}$ is the effective number density, $\sigma(E)$ is the absorption cross-section at photon energy $E, L$ is the sample pathlength, and $\Delta$ is the FWHM value of the spectral broadening that characterizes the spectrometer resolution. The above expression does not account for the pixelation of the CCD array detector explicitly, but instead factors it into the Gaussian spectral broadening function. 


\section{F. DATA ACQUISITION PROCEDURES}

Static photoabsorption spectra are collected by introducing the sample into the gas cell and measuring the transmission of the soft $\mathrm{x}$-ray light. The optical density (absorbance) at photon energy $E$ is given by $\operatorname{OD}(E)=-\log \left[I(E) / I_{0}(E)\right]$, where $I(E)$ is the spectral intensity after the sample, and $I_{0}(E)$ is the spectral intensity without the sample. The CCD camera integration time is typically set to $5 \mathrm{~s}$ for each high-order harmonic spectrum collected. The gas cell is then alternately translated into and out of the soft x-ray beam to record $I(E)$ and $I_{0}(E)$, respectively, with each pair of $I(E)$ and $I_{0}(E)$ spectra constituting one data set. Compared to alternately filling and evacuating the gas cell, translating the gas cell into and out of the beam provides a more efficient way of collecting the static absorption spectrum. Depending on the optical density of the sample, up to 32 sets of high-order harmonic spectra with and without the sample are acquired. These spectra are then averaged to yield the static absorption spectrum, with accompanying error bars computed at the $95 \%$ confidence interval.

Two types of static photoabsorption spectra can be collected. The nonresonant static absorption spectrum arises from the photoionization of valence electrons by the soft x-ray light and is featureless. As such, it can be collected by measuring the change in transmission at the peak of each individual high-order harmonic, resulting in a spectrum with a sampling interval of $\sim 3 \mathrm{eV}$. On the other hand, resonant absorption features in atomic species have a typical linewidth of $\sim 0.1 \mathrm{eV}$. Rather than sampling only at the peak of each high-order harmonic, it becomes necessary to measure the transmission by sampling all the data points within that spectral region. The sampling interval is then dictated by the energy spacing between adjacent pixels in the tangential plane of the CCD 
camera, which is $0.1 \mathrm{eV}$ for the home-built spectrometer. In addition, for narrow atomic resonances, the peak of an individual high-order harmonic is wavelength-tuned to coincide with the absorption maximum of the resonant transition. The higher photon flux available at the harmonic peak enables a resonant absorption spectrum to be obtained by averaging typically four sets of spectra. For broader absorption features, such as those of molecules, absorption spectra can be collected by employing the weak continuum that is present below and between the discrete harmonics. Due to the considerably lower photon flux in the continuum, however, averaging over a larger data set becomes necessary.

Transient absorption spectra are typically obtained by collecting the probe spectrum with the pump beam incident on the sample ("pump-on") and another probe spectrum with the pump beam blocked ("pump-off"). The transient absorption spectrum at photon energy $E$ and time delay $\Delta t$ is subsequently given by $\Delta \mathrm{OD}(E, \Delta t)=-\log \left[I_{O N}(E, \Delta t) / I_{O F F}(E, \Delta t)\right]$, where $I_{O N}(E, \Delta t)$ and $I_{O F F}(E, \Delta t)$ are the spectral intensities of the probe with and without the pump beam, respectively. In our experiment, the presence of stray pump light incident on the detector results in a constant background offset. For a time- and energy-independent background offset of $I_{b}$, the measured transient absorption signal $\Delta \mathrm{OD}_{\text {meas }}(E, \Delta t)$, up to first order in $I_{b} / I_{O N}(E, \Delta t)$, is given by

$$
\begin{aligned}
\Delta \mathrm{OD}_{\text {meas }}(E, \Delta t) & =-\log \left[\frac{I_{O N}(E, \Delta t)+I_{b}}{I_{O F F}(E, \Delta t)}\right] \\
& =\Delta \mathrm{OD}_{0}(E, \Delta t)-\frac{1}{\ln 10} \frac{I_{b}}{I_{O N}(E, \Delta t)},
\end{aligned}
$$


where $\Delta \mathrm{OD}_{0}(E, \Delta t)$ is the transient absorption signal in the absence of the background offset. From Eq. (2), it can be inferred that a background of order $I_{b} / I_{O N}(E, \Delta t) \sim 0.1$ is sufficiently large to yield an error that is comparable to a relatively weak transient absorption signal of $\Delta \mathrm{OD} \sim 0.05$. The background offset therefore precludes the use of $I_{O F F}(E, \Delta t)$ as the reference spectrum; therefore harmonic spectra collected at $\Delta t=-500$ fs are used as reference instead. The transient absorption signal is then given by $\Delta \mathrm{OD}(E, \Delta t)=-\log \left[I_{O N}(E, \Delta t) / I_{O N}(E,-500 \mathrm{fs})\right]$. Note that a negative time delay implies that the probe pulse arrives at the sample before the pump pulse.

Transient absorption spectra at a fixed time delay $\Delta t$ are obtained by collecting the high-order harmonic spectra with the delay stage alternating between -500 fs and $\Delta t$; each pair of harmonic spectra collected in this manner would yield one transient absorption spectrum at $\Delta t$. This procedure minimizes the effect of long-term drift of the high-order harmonics. The CCD integration time is set to $2 \mathrm{~s}$ for the acquisition of each harmonic spectrum, corresponding to an acquisition of 2000 individual laser pulses. Synchronization between the computer-controlled delay stage and the CCD camera is achieved by triggering the CCD camera with a TTL output from a multifunction data acquisition card (National Instruments, PCI-6221) after the delay stage moves to its preset position (time delay). Transient absorption spectra are typically obtained by averaging 128 individual spectra.

Pump-probe time traces are obtained by collecting the high-order harmonic spectrum over a CCD integration time of $2 \mathrm{~s}$ at each time delay as the delay stage is scanned from negative $(\Delta t<0)$ to positive $(\Delta t>0)$ time. The $\Delta \mathrm{OD}$ at each time delay is then computed by integrating the number of counts over a typical spectral bandwidth of 0.1 
$\mathrm{eV}$, and using the corresponding value obtained at $-500 \mathrm{fs}$ time delay as reference. Typically eight sets of such time traces are then averaged to yield the pump-probe time traces. Reported error bars for both transient absorption spectra and pump-probe time traces correspond to $95 \%$ confidence interval limits.

\section{III.RESULTS AND DISCUSSION}

Keeping the goal in mind of employing high-order harmonics in transient absorption spectroscopy, results from the characterization of the high-order harmonics are presented in this section. Characterization includes determining the wavelength-tunability of the high-order harmonics, as well as measuring the photon flux and the spatial beam profile of the soft x-ray light. In addition, examples of both static and transient photoabsorption spectra obtained with the apparatus are provided.

\section{A. HIGH-ORDER HARMONIC SPECTRA}

Typical high-order harmonic spectra obtained with $\mathrm{Ar}$ and $\mathrm{Ne}$ as the medium are shown in Figs. 6a and 6b, respectively. Note that the peaks below 37 eV in Fig. $6 \mathrm{~b}$ arise from the second-order diffraction of the grating. Due to plasma-induced blueshifting of the fundamental $\operatorname{light}^{49}$ in the capillary waveguide (see below), both $\mathrm{Ar}$ and $\mathrm{Ne}$ harmonics exhibit peak spacings that are greater than the $3.1 \mathrm{eV}$ spacing that is expected from use of a $800 \mathrm{~nm}$ HHG driver pulse. The Ar harmonics show peak spacings of 3.24 $\mathrm{eV}$ (black line) and $3.40 \mathrm{eV}$ (grey line), whereas the Ne harmonics show a peak spacing of $3.26 \mathrm{eV}$. 
Two sets of high-order harmonic spectra are shown for Ar. The spectrum with its peak at $39.5 \mathrm{eV}$ (25th harmonic, black line) is generated with an Ar inlet pressure of 50 Torr and a NIR input pulse energy of $1.0 \mathrm{~mJ}$, whereas the spectrum with its peak at 43.5 $\mathrm{eV}$ (27th harmonic, grey line) is generated at the same inlet pressure, but with $1.4 \mathrm{~mJ}$ of input pulse energy. The latter exhibits peaks with bandwidths of $1.0 \mathrm{eV}$ FWHM, whereas the bandwidth of the individual harmonics in the former spectrum is $0.4 \mathrm{eV}$. Since both spectra are collected under identical spectral resolution and spatial beam quality conditions, the large bandwidth observed for the Ar harmonics produced with $1.4 \mathrm{~mJ}$ of input pulse energy and an inlet pressure of 50 Torr is not an artifact.

Inspecting the spectrum of the NIR driving pulse collected after the exit of the capillary waveguide provides a possible explanation for the origin of the large bandwidth observed. When Ar is introduced, blueshifting of the fundamental light occurs due to laser-induced plasma formation ${ }^{49}$ in the capillary waveguide (see below). This is evidenced from the spectra of the residual HHG driver pulse obtained by translating the broadband $800 \mathrm{~nm}$ high reflector (mounted on the push-pull) into the beam path after the exit of the capillary waveguide (Fig. 7). An increase in the plasma density at higher input pulse energies leads to a larger degree of spectral blueshifting. The blueshifting of the fundamental light is accompanied by a dramatic increase in its spectral bandwidth, thereby resulting in large spectral bandwidths for the individual high-order harmonics generated under such conditions. Note that plasma-induced blueshifting of the NIR driver pulse also explains the $0.8 \mathrm{eV}$ shift between the two harmonic spectra.

Finally, the high-order harmonics generated with a $\mathrm{Ne}$ inlet pressure of 80 Torr and an input pulse energy of $1.8 \mathrm{~mJ}$ are observed to extend to the $\mathrm{Al} L_{3}$-edge cut-off. The 
bandwidth of each individual harmonic is $0.7 \mathrm{eV}$. It is interesting to note that a continuum component is visible underneath the set of discrete harmonic peaks. The ratio of harmonic peak amplitude to the continuum amplitude is $\sim 20: 1$. This weak continuum underlying the discrete harmonic peaks allows, in principle, acquisition of the entire absorption spectrum without the need to tune the harmonic photon energies. However, from signal-to-noise considerations, it is desirable for the peak of a harmonic to coincide with the (static or transient) absorption maximum of a particular atom/molecule, especially when a narrow atomic transition is being probed. This requires the peak of the individual harmonics to be wavelength-tunable, which is discussed in the following section.

\section{B. WAVELENGTH-TUNING BY PLASMA-INDUCED BLUESHIFTING}

Wahlström et al. first demonstrated the wavelength-tuning of high-order harmonics by varying the peak intensity of the NIR driving pulse or the number density in an Ar or Xe gas jet. ${ }^{49}$ A recent study showed that wavelength-tuning of high-order harmonics generated in an Ar-filled capillary waveguide can be induced by varying the chirp of the NIR driving pulse. ${ }^{50}$ In both cases, wavelength-tuning is due to blueshifting of the fundamental driver pulse in a plasma medium, given by ${ }^{49}$

$$
\delta E_{f}=\frac{h^{2} N}{2 \pi m_{e} c} \frac{1}{E_{f}} \frac{d f_{e}}{d t} L,
$$

where $h$ is Planck's constant, $N$ is the number density (proportional to gas pressure), $m_{e}$ is the free electron mass, $c$ is the speed of light in vacuum, $E_{f}$ is the photon energy of the 
fundamental, $d f_{e} / d t$ is the rate of increase of the ionization fraction, and $L$ is the pathlength. The corresponding blueshift of the $q$ th harmonic is therefore given by $\delta E_{q}=q \delta E_{f}$. Here we demonstrate that plasma blueshifting of the fundamental in a Nefilled capillary waveguide can conveniently be used to tune the photon energies of highorder harmonics output, even though the ionization potential of $\mathrm{Ne}(21.56 \mathrm{eV})$, which affects $d f_{e} / d t$, is considerably higher than that of $\operatorname{Ar}(15.76 \mathrm{eV})$ or $\mathrm{Xe}(12.13 \mathrm{eV})$, resulting in a substantially lower plasma density being created. Fig. 8a shows the linear increase in photon energy for the various harmonics as a function of the capillary inlet pressure, as expected from the linear relationship between $\delta E_{q}$ and $N$ in Eq. (3). Furthermore, the degree of plasma blueshifting per unit pressure should be linearly proportional to the harmonic order $q$. This linear relationship is observed in Fig. $8 \mathrm{~b}$, which verifies that the observed wavelength-tuning is indeed due to plasma blueshifting of the fundamental light. Note that aside from the plasma-induced blueshifting of the fundamental light in the capillary yielding the blueshifted high-order harmonics, the highorder harmonics themselves can also undergo plasma-induced blueshifting. In the case of the latter, however, the extent of blueshifting per unit pressure as a function of harmonic order would exhibit a $1 / q$-dependence (since $\delta E_{q} \propto 1 / E_{q}=1 / q E_{f}$ ).

\section{PHOTON FLUX MEASUREMENTS}

By employing a calibrated soft x-ray photodiode, the wavelength-integrated photon flux of the Ar harmonics at an inlet pressure of 40 Torr and a HHG driver pulse energy of $1 \mathrm{~mJ}$ is found to be $1 \times 10^{9}$ photons/pulse at the source. This corresponds to a conversion

efficiency of $2 \times 10^{-7}$ from the fundamental light. At an average photon energy of $40 \mathrm{eV}$ 
and a repetition rate of $1 \mathrm{kHz}$, the estimated $\mathrm{HHG}$ pulse energy is $6 \mathrm{~nJ}$ and the average output power is $6 \mu \mathrm{W}$. This photon flux is comparable to the previously reported wavelength-integrated photon flux of $4 \times 10^{8}$ photons/pulse for the harmonics centered around $31 \mathrm{eV} .^{51}$ At a slightly lower harmonic order, a photon flux of $1.4 \times 10^{9}$ photons/pulse was measured for the 15th harmonic generated in an Ar-filled capillary waveguide. $^{52}$

In addition to using the soft $\mathrm{x}$-ray photodiode, an independent estimate of the photon flux can be obtained from the number of counts registered within a preset spectral bandwidth by the soft x-ray CCD camera. This procedure requires knowledge of the transmittance and the reflectance of the individual optical components in the beam path. The reflectivity curve of the gold-coated toroidal mirrors is obtained from the Center for X-Ray Optics (Lawrence Berkeley National Laboratory) website. ${ }^{40}$ The transmission curve of the $\mathrm{Al}$ foil is obtained from a measurement performed at $\mathrm{BL} 6.3 .2$ of the Advanced Light Source. An efficiency of 5\% is assumed for the diffraction grating. The quantum efficiency of the soft x-ray CCD camera remains approximately constant at $\sim 40 \%$ over the wavelength range of $30-100 \mathrm{eV}$. With these parameters, the photon flux is calculated for the harmonic with the highest amplitude in each spectrum shown in Figs. $6 \mathrm{a}$ and $6 \mathrm{~b}$. The results are summarized in Table 1. The three-orders of magnitude decrease in photon flux for the Ne harmonic compared to the Ar harmonic is significantly larger than the typical two orders of magnitude decrease observed in previous studies in which phase-matched HHG was achieved. ${ }^{53,54}$ The origin of the observed discrepancy is unclear. One possibility to consider is that the most intense Ne harmonic is produced at a photon energy beyond the $L_{3}$-edge cut-off of the $\mathrm{Al}$ filters. Finally, it is important to note 
that the available photon flux of $\sim 10^{3}$ photons/pulse (over a spectral bandwidth of $2 \mathrm{eV}$ ) in the continuum of the Ne harmonics is already sufficient for performing both static and transient absorption spectroscopy (see below).

\section{SPATIAL BEAM PROFILE MEASUREMENTS}

The focal spot sizes of the different high-order harmonics at the interaction region are measured for different Ar and Ne inlet pressures using the scanning knife-edge method. Typical knife-edge scan traces are shown in Figs. 9a and 9b. The high spatial beam quality obtained from the $8 \mathrm{~cm}$ long capillary waveguide agrees with previous results obtained from fringe visibility measurements, which show that capillary waveguides with lengths greater than $6 \mathrm{~cm}$ yield soft x-ray beams with full spatial coherence. ${ }^{55}$

In addition to the focal spot size, the wavelength-integrated beam profile of the highorder harmonics in the far-field is recorded by positioning a soft x-ray CCD camera 30 $\mathrm{cm}$ after the sample focus. Typical beam profiles for the Ar and $\mathrm{Ne}$ harmonics are shown in Figs. 10a and 10b, respectively; these images are collected with 50 Torr of Ar at 1.0 $\mathrm{mJ}$ of input pulse energy and 40 Torr of $\mathrm{Ne}$ at $1.8 \mathrm{~mJ}$ of input pulse energy. The estimated half-angle divergences for the Ar harmonics are $1.3 \mathrm{mrad}$ and $1.5 \mathrm{mrad}$ in the horizontal and vertical direction, respectively. The spatial profile of the Ne harmonics exhibit a broad background, which upon subtraction, yields estimated half-angle divergences of $0.6 \mathrm{mrad}$ and $0.7 \mathrm{mrad}$ in the horizontal and vertical directions, respectively. 


\section{E. STATIC PHOTOABSORPTION SPECTROSCOPY}

High-order harmonic spectra recorded with and without Xe gas in the sample gas cell are shown in Fig. 11a. The resultant absorption spectrum, obtained by evaluating the optical density at the peak of each individual harmonic, is shown in Fig. 11b. Due to the $\sim 3 \mathrm{eV}$ sampling interval, the spectrum reveals only the nonresonant absorption component. The low energy side of the spectrum rises to the valence photoionization region, whereas the increase in absorption cross-section at higher photon energies is due to the $4 \mathrm{~d} \rightarrow \varepsilon f$ shape resonance that peaks at $\sim 100 \mathrm{eV} .{ }^{56}$ Comparison of the measured optical densities to the absorption cross-sections reported in the literature ${ }^{57}$ enables the sample gas pressure to be inferred, since $n_{\text {eff }}=\mathrm{OD}_{\text {meas }}(E) \ln 10 / \sigma(E) L$, where $n_{\text {eff }}$ is the effective number density (see Section IID), $\mathrm{OD}_{\text {meas }}(E)$ is the measured optical density, $\sigma(E)$ is the absorption cross-section, and $L$ is the nominal sample path length. For this set of data, the number density corresponds to a sample pressure $51 \pm 1$ Torr.

In addition to the nonresonant absorption, close inspection of Fig. 11a shows the presence of dips at $65.1 \mathrm{eV}$ and $68.3 \mathrm{eV}$ in the high-order harmonic spectrum collected in the presence of Xe. These dips are indicative of resonant absorption features. The corresponding absorption spectra in the range of $64.6 \mathrm{eV}-65.6 \mathrm{eV}$ and $67.8 \mathrm{eV}-68.8$ $\mathrm{eV}$ are shown in Figs. 11c and 11d, respectively. The absorption feature at $65.1 \mathrm{eV}$

corresponds to the ${ }^{1} S_{0} \rightarrow 4 d^{-1}\left({ }^{2} D_{5 / 2}\right) 6 p\left({ }^{2} P_{3 / 2}\right)$ transition, whereas that at $68.3 \mathrm{eV}$ corresponds to the ${ }^{1} S_{0} \rightarrow 4 d^{-1}\left({ }^{2} D_{3 / 2}\right) 7 p\left({ }^{2} P_{1 / 2}\right)$ transition. The absorption cross-section for these transitions can be decomposed into resonant and nonresonant absorption components 


$$
\sigma(E)=\sigma_{R} \frac{(\Gamma / 2)^{2}}{\left(E-E_{0}\right)^{2}+(\Gamma / 2)^{2}}+\sigma_{N R}(E)
$$

In the above expression, the resonant absorption feature is a Lorentzian centered at $E_{0}$ with a peak absorption cross-section of $\sigma_{R}$ and $\Gamma$ is the natural linewidth. The nonresonant absorption cross-section is $\sigma_{N R}(E)$. Note that, in principle, the interference between the direct photoionization and autoionization channels accompanying these atomic core-level transitions requires the resonant absorption feature to be fit to a FanoBeutler lineshape ${ }^{58}$ given by

$$
\sigma_{F B}(\varepsilon)=\sigma_{R} \frac{1+\rho^{2}\left(q^{2}-1+2 q \varepsilon\right)}{1+\varepsilon^{2}},
$$

where $\varepsilon=2\left(E-E_{0}\right) / \Gamma$ is the reduced energy variable, $\rho=\left\langle\Psi_{d} \mid \Psi_{a}\right\rangle$ is the overlap between the continuum states produced by direct photoionization and autoionization, and $q$ is the Fano lineshape parameter. ${ }^{59}$ For the resonant $4 d$ photoabsorption of Xe, however, the direct transition yields singly excited $\mathrm{Xe}^{+}$ions, whereas autoionization of the coreexcited state yields doubly excited $\mathrm{Xe}^{+}$ions. ${ }^{58}$ Due to poor overlap between these states, $\rho \rightarrow 0$ and the Fano-Beutler lineshape can be approximated by a Lorentzian lineshape. The large lifetime broadening of these core-level transitions allows the neglect of other line broadening mechanisms such as power broadening, transit broadening, pressure broadening, and Doppler broadening.

From the fits to the resonant absorption lineshapes (Figs. 11c and 11d), the location of the transition maxima are determined to be $65.06 \pm 0.01 \mathrm{eV}$ and $68.30 \pm 0.01 \mathrm{eV}$. For the $65.1 \mathrm{eV}$ transition, the resonant absorption cross-section is $(14.0 \pm 1.2) \times 10^{-18} \mathrm{~cm}^{2}$ and the nonresonant absorption cross-section is $(1.8 \pm 0.1) \times 10^{-18} \mathrm{~cm}^{2}$. By fixing the natural 
linewidth to the literature value of $109.8 \mathrm{meV},{ }^{60}$ the FWHM value that characterizes the spectrometer resolution is found to be $\Delta=0.20 \pm 0.01 \mathrm{eV}$, in accord with the design specification. The absorption maximum and resonant absorption cross-section are in good agreement with the literature value of $E_{0}=65.11 \mathrm{eV}$ and $\sigma_{R}=(13.6 \pm 0.8) \times 10^{-18}$ $\mathrm{cm}^{2}{ }^{61,62}$ The nonresonant absorption cross-section is significantly larger than the literature value of $(1.04 \pm 0.06) \times 10^{-18} \mathrm{~cm}^{2},{ }^{62}$ but in good agreement with the value of $1.7 \times 10^{-18} \mathrm{~cm}^{2}$ obtained from linear interpolation of inner-shell electron energy loss spectral data. ${ }^{57}$ Note that the variation in $\sigma_{N R}$ over the spectral range shown in Fig. $11 \mathrm{c}$ is not easily discernible, thereby allowing $\sigma_{N R}$ to be fit to a constant value in the fitting routine. On the other hand, the significant variation in $\sigma_{N R}$ with photon energy for the $68.3 \mathrm{eV}$ transition can be attributed to the broad $4 d \rightarrow \varepsilon f$ shape resonance present beneath the resonant absorption feature. For this transition, the resonant absorption cross-section is $(1.5 \pm 0.2) \times 10^{-18} \mathrm{~cm}^{2}$, the nonresonant absorption cross-section is $(2.9 \pm 0.4) \times 10^{-18}$ $\mathrm{cm}^{2}$ (at $\left.68.3 \mathrm{eV}\right)$, and the slope of the nonresonant absorption cross-section is $(0.30 \pm$ $0.07) \times 10^{-18} \mathrm{~cm}^{2} / \mathrm{eV}$. The absorption maximum and nonresonant absorption crosssection are both in good agreement with literature values of $E_{0}=68.34 \mathrm{eV}$ and $\sigma_{N R}=2.6$ $\times 10^{-18} \mathrm{~cm}^{2}{ }^{61,57}$ The latter is obtained from linear interpolation of inner-shell electron energy loss data, which also yields a nonresonant absorption cross-section slope of $0.22 \times$ $10^{-18} \mathrm{~cm}^{2} / \mathrm{eV}$. The ratio of the peak areas after subtraction of the nonresonant background is $A\left(4 d_{5 / 2}^{-1} 6 p_{3 / 2}\right): A\left(4 d_{3 / 2}^{-1} 7 p_{1 / 2}\right)=6.1 \pm 2.2$, in good agreement with the ratio of oscillator strengths $f\left(4 d_{5 / 2}^{-1} 6 p_{3 / 2}\right): f\left(4 d_{3 / 2}^{-1} 7 p_{1 / 2}\right)=5.5 \pm 2.4$ reported in the literature. ${ }^{61}$ These results demonstrate the ability to use high-order harmonics to perform quantitative photoabsorption spectroscopy. 
Aside from observing narrow atomic transitions, the experimental apparatus can also be used to measure the photoabsorption spectra of species that exhibit broad spectral features. For broad transitions, the continuum component beneath the discrete harmonic peaks can be utilized to measure the absorption spectrum. Fig. 12a shows the high-order harmonic spectra obtained with an evacuated gas cell and when the gas cell is filled with $17 \pm 1$ Torr of $\mathrm{CH}_{2} \mathrm{Br}_{2}$. Comparison of the two spectra suggests the presence of a resonant absorption feature in the continuum region at $70.5 \mathrm{eV}$ in addition to the nonresonant absorption present across the entire measured energy range. The resultant absorption spectrum, obtained by averaging over 32 sets of spectra, is shown in Fig. 12b. The broad absorption peak has a FWHM of $\sim 2 \mathrm{eV}$, which can be fit to a superposition of two Gaussian peaks centered at $70.5 \mathrm{eV}$ and $71.5 \mathrm{eV}$. In this energy region, the observed transitions correspond to the promotion of an electron in the $\mathrm{Br} 3 d$ core-level to a $\sigma^{*}(\mathrm{C}-$ Br) anti-bonding orbital. The dissociative nature of the core hole-excited state gives rise

to a broad absorption resonance. ${ }^{63,64}$ The $1 \mathrm{eV}$ spacing between the two peaks is consistent with the spin-orbit splitting of the $\mathrm{Br} 3 d$ core level. ${ }^{65}$ The lower energy component is broader (1.5 eV FWHM) than the higher energy component $(1.1 \mathrm{eV}$ FWHM), in accord with the effect of ligand-field splitting on the $\mathrm{Br} 3 d$ core levels. ${ }^{64}$

\section{F. TRANSIENT ABSORPTION SPECTROSCOPY}

This instrument has already been used to investigate the interaction of an intense laser pulse with atoms and molecules in the strong-field regime. In the strong-field regime, the electric field of the laser pulse induces a significant distortion of the atomic potential as the field becomes comparable to the effective potential experienced by a valence electron 
due to the ion core and other electrons. ${ }^{19,66}$ The onset of this regime typically occurs at electric field strengths of $\sim 1 \times 10^{8} \mathrm{~V} / \mathrm{cm}$, corresponding to laser peak intensities of $\sim 1 \times$ $10^{13} \mathrm{~W} / \mathrm{cm}^{2}$. Here the sequential ionization of $\mathrm{Xe}$ atoms to form $\mathrm{Xe}^{2+}$ is used to demonstrate the ability of this apparatus to achieve sub-15-fs time resolution when used in strong-field ionization studies. Fig. 13 shows the time-evolution of the high-order harmonic centered at $57.0 \mathrm{eV}$ as the $\mathrm{Xe}$ sample gas target $(15 \pm 1$ Torr $)$ is irradiated by an $800 \mathrm{~nm}$ laser pulse with a peak intensity of $6.2 \times 10^{14} \mathrm{~W} / \mathrm{cm}^{2}$. Each spectrum is obtained from the average of 8 individual high-order harmonic spectra. A decrease in the spectral intensity at $57.0 \mathrm{eV}$ as the delay stage is scanned from negative to positive time delay is indicative of a transient absorption feature at that photon energy. The transient absorption spectrum shown in Fig. 14 is obtained from the average of 8 sets of individual transient absorption spectra collected between $100 \mathrm{fs}-500 \mathrm{fs}$; since a total of 12 time points are sampled over this time delay range, the spectrum is obtained from the average of 96 individual transient absorption spectra. In addition to the $\mathrm{Xe}^{+2} P_{3 / 2}\left(5 p^{-1}\right) \rightarrow{ }^{2} D_{5 / 2}\left(4 d^{-1}\right)$ and ${ }^{2} P_{1 / 2}\left(5 p^{-1}\right) \rightarrow{ }^{2} D_{3 / 2}\left(4 d^{-1}\right)$ transitions located at $55.4 \mathrm{eV}$ and $56.1 \mathrm{eV}$, respectively, ${ }^{48}$ a prominent absorption feature is observed at $57.0 \mathrm{eV}$. This absorption line actually consists of two unresolved $\mathrm{Xe}^{2+}$ absorption peaks - the ${ }^{3} P_{2}\left(5 p^{-2}\right) \rightarrow{ }^{1} D_{2}\left(4 d^{-1} 5 p^{-1}\right)$ transition at $56.94 \mathrm{eV}$ and the ${ }^{3} P_{2}\left(5 p^{-2}\right) \rightarrow{ }^{3} D_{3}\left(4 d^{-1} 5 p^{-1}\right)$ transition at $57.11 \mathrm{eV} .{ }^{48}$ Furthermore, it is interesting to note that the two $\mathrm{Xe}^{+}$transient absorption peaks are visible even though the high-order harmonic light in that spectral region consists of only the weak continuum.

Finally, the time trace obtained from monitoring the spectral intensity at $57.0 \mathrm{eV}$ as a function of time delay is shown in Fig. 15. This trace is obtained from the average of 8 
individual time traces. The step-like growth of the signal originates from the crosscorrelation of the high-order harmonic pulse duration with the increase in $\mathrm{Xe}^{2+}$ yield within the $800 \mathrm{~nm}$ pump pulse envelope, which can be fit to a convolution of a step function with a Gaussian function. The FWHM value of the Gaussian function is $13 \pm 3$ fs. Note that this value sets the upper-bound for the pulse duration of the soft x-ray light. As such, the achievable time resolution for an experiment employing a single-photon excitation pump step is currently limited by the $\sim 45 \mathrm{fs}$ pulse duration of the $800 \mathrm{~nm}$ light. The use of a sub-10-fs optical pump pulse, such as those produced by a noncollinear optical parametric amplifier ${ }^{67}$ or its frequency-upconverted output, ${ }^{68}$ should enable access to the study of ultrafast molecular dynamics occurring on the 10 fs time scale. Nevertheless, the experimental apparatus in its current configuration can already be used to address strong-field ionization phenomena, such as strong-field dissociative photoionization, ${ }^{69-71}$ with sub-15-fs time resolution.

\section{IV.SUMMARY}

A detailed description of a table-top, laser-based instrument for performing femtosecond time-resolved soft x-ray transient absorption spectroscopy is provided in this paper. The instrument is based on an amplified femtosecond Ti:sapphire laser system and produces ultrashort soft x-ray pulses by high-order harmonic generation. By employing a capillary waveguide filled with $\mathrm{Ne}$, high-order harmonics up to the $\mathrm{Al} L_{3^{-}}$ edge $(72.64 \mathrm{eV})$ are produced, which enables access to the probing of core levels. Wavelength-tuning of the Ne harmonics by means of plasma-induced blueshifting of the fundamental light is observed. The photon flux of the Ne harmonics is approximately 
three orders of magnitude lower than that of the Ar harmonics, although their spatial beam qualities are comparable. Static photoabsorption measurements that probe the $4 d$ core level of Xe yield nonresonant and resonant absorption cross-sections that are in reasonably good agreement with those reported in the literature. Finally, transient absorption measurements on the sequential double ionization of $\mathrm{Xe}$ to produce $\mathrm{Xe}^{2+}$ give an estimated time resolution of $13 \pm 3$ fs for this instrument. This table-top instrument should therefore enable the core-level absorption probing of ultrafast dynamics accompanying strong-field ionization with sub-15-fs time resolution.

\section{ACKNOWLEDGMENTS}

We are greatly indebted to A. Paul and Profs. M. M. Murnane and H. C. Kapteyn for their assistance in implementing the HHG setup. We thank Y. Liu for measuring the transmission curve of the Al foil and T. Pfeifer for useful discussions. This work was supported by the NSF ERC for Extreme Ultraviolet Science and Technology (EEC0310717) and the LDRD program at LBNL, with additional equipment support from DOE (DE-AC02-05CH11351). 


\section{REFERENCES}

1. C. Bressler and M. Chergui, Chem. Rev. 104, 1781 (2004).

2. L. X. Chen, Annu. Rev. Phys. Chem. 56, 221 (2005).

3. T. Pfeifer, C. Spielmann, and G. Gerber, Rep. Prog. Phys. 69, 443 (2006).

4. A. Rousse, C. Rischel, and J. C. Gauthier, Rev. Mod. Phys. 73, 17 (2001).

5. L. X. Chen, W. J. H. Jäger, G. Jennings, D. J. Gosztola, A. Munkholm, and J. P. Hessler, Science 292, 262 (2001).

6. M. Saes, C. Bressler, R. Abela, D. Grolimund, S. L. Johnson, P. A. Heimann, and M. Chergui, Phys. Rev. Lett. 90, 047403 (2003).

7. S. L. Johnson, P. A. Heimann, A. M. Lindenberg, H. O. Jeschke, M. E. Garcia, Z. Chang, R. W. Lee, J. J. Rehr, and R. W. Falcone, Phys. Rev. Lett. 91, 157403 (2003).

8. R. W. Schoenlein, S. Chattopadhyay, H. H. W. Chong, T. E. Glover, P. A. Heimann, C. V. Shank, A. A. Zholents, and M. S. Zolotorev, Science 287, 2237 (2000).

9. A. Cavalleri, M. Rini, H. H. W. Chong, S. Fourmaux, T. E. Glover, P. A. Heimann, J. C. Kieffer, and R. W. Schoenlein, Phys. Rev. Lett. 95, 067405 (2005).

10. A. Cavalleri, S. Wall, C. Simpson, E. Statz, D. W. Ward, K. A. Nelson, M. Rini, and R. W. Schoenlein, Nature 442, 664 (2006).

11. A. M. Lindenberg, J. Larsson, K. Sokolowski-Tinten, K. J. Gaffney, C. Blome, O. Synnergren, J. Sheppard, C. Caleman, A. G. MacPhee, D. Weinstein, D. P. Lowney, T. K. Allison, T. Matthews, R. W. Falcone, A. L. Cavalieri, D. M. Fritz, S. H. Lee, P. H. Bucksbaum, D. A. Reis, J. Rudati, P. H. Fuoss, C. C. Kao, D. P. Siddons, R. Pahl, J. Als-Nielsen, S. Duesterer, R. Ischebeck, H. Schlarb, H. Schulte-Schrepping, Th. 
Tschentscher, J. Schneider, D. von der Linde, O. Hignette, F. Sette, H. N. Chapman, R. W. Lee, T. N. Hansen, S. Techert, J. S. Wark, M. Bergh, G. Huldt, D. van der Spoel, N. Timneanu, J. Hajdu, R. A. Akre, E. Bong, P. Krejcik, J. Arthur, S. Brennan, K. Luening, and J. B. Hastings, Science 308, 392 (2005).

12. D. M. Fritz, D. A. Reis, B. Adams, R. A. Akre, J. Arthur, C. Blome, P. H. Bucksbaum, A. L. Cavalieri, S. Engemann, S. Fahy, R. W. Falcone, P. H. Fuoss, K. J. Gaffney, M. J. George, J. Hajdu, M. P. Hertlein, P. B. Hillyard, M. Horn-von Hoegen, M. Kammler, J. Kaspar, R. Kienberger, P. Krejcik, S. H. Lee, A. M. Lindenberg, B. McFarland, D. Meyer, T. Montagne, É. D. Murray, A. J. Nelson, M. Nicoul, R. Pahl, J. Rudati, H. Schlarb, D. P. Siddons, K. Sokolowski-Tinten, Th. Tschentscher, D. von der Linde, and J. B. Hastings, Science 315, 633 (2007).

13. H. Wabnitz, L. Bittner, A. R. B. de Castro, R. Döhrmann, P. Gürtler, T. Laarmann, W. Laasch, J. Schulz, A. Swiderski, K. von Haeften, T. Möller, B. Faatz, A. Fateev, J. Feldhaus, C. Gerth, U. Hahn, E. Saldin, E. Schneidmiller, J. Sytchev, K. Tiedtke, R. Treusch, and M. Yurkov, Nature 420, 482 (2002).

14. F. Ráksi, K. R. Wilson, Z. M. Jiang, A. Ikhlef, C. Y. Côté, and J.-C. Kieffer, J. Chem. Phys. 104, 6066 (1996).

15. C. Rose-Petruck, R. Jimenez, T. Guo, A. Cavalleri, C. W. Siders, F. Ráksi, J. A. Squier, B. C. Walker, K. R. Wilson, and C. P. J. Barty, Nature 398, 310 (1999).

16. K. Sokolowski-Tinten, C. Blome, J. Blums, A. Cavalleri, C. Dietrich, A. Tarasevitch, I. Uschmann, E. Förster, M. Kammler, M. Horn-von-Hoegen, and D. von der Linde, Nature 422, 287 (2003). 
17. M. Bargheer, N. Zhavoronkov, Y. Gritsai, J. C. Woo, D. S. Kim, M. Woerner, and T. Elsaesser, Science 306, 1771 (2004).

18. T. Lee, Y. Jiang, C. G. Rose-Petruck, and F. Benesch, J. Chem. Phys. 122, 084506 (2005).

19. T. Brabec and F. Krausz, Rev. Mod. Phys. 72, 545 (2000).

20. H. C. Kapteyn, M. M. Murnane, and I. P. Christov, Phys. Today 58, 39 (2005).

21. L. Nugent-Glandorf, M. Scheer, D. A. Samuels, A. M. Mulhisen, E. R. Grant, X. M. Yang, V. M. Bierbaum, and S. R. Leone, Phys Rev. Lett. 87, 193002 (2001).

22. D. Strasser, F. Goulay, and S. R. Leone, J. Chem. Phys. 127, 184305 (2007).

23. P. Siffalovic, M. Drescher, M. Spieweck, T. Wiesenthal, Y. C. Lim, R. Weidner, A. Elizarov, and U. Heinzmann, Rev. Sci. Instrum. 72, 30 (2001).

24. M. Bauer, C. Lei, K. Read, R. Tobey, J. Gland, M. M. Murnane, and H. C. Kapteyn, Phys. Rev. Lett. 87, 025501 (2001).

25. L. Miaja-Avila, C. Lei, M. Aeschlimann, J. L. Gland, M. M. Murnane, H. C. Kapteyn, and G. Saathoff, Phys. Rev. Lett. 97, 113604 (2006).

26. M. Hentschel, R. Kienberger, Ch. Spielmann, G. A. Reider, N. Milosevic, T. Brabec, P. Corkum, U. Heinzmann, M. Drescher, and F. Krausz, Nature 414, 509 (2001).

27. M. Drescher, M. Hentschel, R. Kienberger, M. Uiberacker, V. Yakovlev, A. Scrinzi, T. Westerwalbesloh, U. Kleineberg, U. Heinzmann, and F. Krausz, Nature 419, 803 (2002).

28. M. Uiberacker, Th. Uphues, M. Schultze, A. J. Verhoef, V. Yakovlev, M. F. Kling, J. Rauschenberger, N. M. Kabachnik, H. Schöder, M. Lezius, K. L. Kompa, M. J. J. 
Vrakking, S. Hendel, U. Kleineberg, U. Heinzmann, M. Drescher, and F. Krausz, Nature 446, 627 (2007).

29. A. L. Cavalieri, N. Müller, Th. Uphues, V. S. Yakovlev, A. Baltuška, B. Horvath, B. Schmidt, L. Blümel, R. Holzwarth, S. Hendel, M. Drescher, U. Kleineberg, P. M. Echenique, R. Kienberger, F. Krausz, and U. Heinzmann, Nature 449, 1029 (2007).

30. R. Berlasso, C. Dallera, F. Borgatti, C. Vozzi, G. Sansone, S. Stagira, M. Nisoli, G. Ghiringhelli, P. Villoresi, L. Poletto, M. Pascolini, S. Nannarone, S. De Silvestri, and L. Braicovich, Phys. Rev. B 73, 115101 (2006).

31. E. Seres, J. Seres, and C. Spielmann, Appl. Phys. Lett. 89, 181919 (2006).

32. Z.-H. Loh, M. Khalil, R. E. Correa, R. Santra, C. Buth, and S. R. Leone, Phys. Rev. Lett. 98, 143601 (2007).

33. Z.-H. Loh, C. H. Greene, and S. R. Leone, Chem. Phys., in press.

34. Z.-H. Loh and S. R. Leone, submitted.

35. E. Seres and C. Spielmann, Appl. Phys. Lett. 91, 121919 (2007).

36. P. Villoresi, Appl. Opt. 38, 6040 (1999).

37. L. Poletto, P. Villoresi, E. Benedetti, F. Ferrari, S. Stagira, G. Sansone, and M. Nisoli, Opt. Lett. 32, 2897 (2007); see also erratum: Opt. Lett. 33, 140 (2008).

38. P. A. Lee, P. H. Citrin, P. Eisenberger, and B. M. Kincaid, Rev. Mod. Phys. 53, 769 (1981).

39. C. Iaconis and I. A. Walmsley, Opt. Lett. 23, 792 (1998).

40. http://www-cxro.lbl.gov 
41. A. Rundquist, C. G. Durfee, III, Z. Chang, C. Herne, S. Backus, M. M. Murnane, and H. C. Kapteyn, Science 280, 1412 (1998).

42. R. A. Bartels, A. Paul, H. Green, H. C. Kapteyn, M. M. Murnane, S. Backus, I. P. Christov, Y. W. Liu, D. Attwood, and C. Jacobsen, Science 297, 376 (2002).

43. E. A. J. Marcatili and R. A. Schmeltzer, Bell Syst. Tech. J. 43, 1783 (1964).

44. R. L. Abrams, IEEE J. Quantum Elect. 8, 838 (1972).

45. A. J. Paul, Ph.D. Thesis, University of Colorado, Boulder, 2007.

46. T. Pfeifer, R. Kemmer, R. Spitzenpfeil, D. Walter, C. Winterfeldt, G. Gerber, and C. Spielmann, Opt. Lett. 30, 1497 (2005).

47. J. Seely and B. Kjornrattanawanich, Appl. Opt. 42, 6374 (2003).

48. P. Andersen, T. Andersen, F. Folkmann, V. K. Ivanov, H. Kjeldsen, and J. B. West, J. Phys. B 34, 2009 (2001).

49. C.-G. Wahlström, J. Larsson, A. Persson, T. Starczewski, S. Svanberg, P. Salières, Ph. Balcou, and A. L'Huillier, Phys. Rev. A 48, 4709 (1993).

50. C. A. Froud, E. T. F. Rogers, D. C. Hanna, W. S. Brocklesby, M. Praeger, A. M. de Paula, J. J. Baumberg, and J. G. Frey, Opt. Lett. 31, 374 (2006).

51. R. A. Bartels, A. Paul, H. Green, H. C. Kapteyn, M. M. Murnane, S. Backus, I. P. Christov, Y. Liu, D. Attwood, and C. Jacobsen, Science 297, 376 (2002).

52. E. Constant, D. Garzella, P. Breger, E. Mével, Ch. Dorrer, C. Le Blanc, F. Salin, and P. Agostini, Phys. Rev. Lett. 82, 1668 (1999).

53. M. Schnürer, Z. Cheng, M. Hentschel, G. Tempea, P. Kálmán, T. Brabec, and F. Krausz, Phys. Rev. Lett. 83, 722 (1999). 
54. J.-F. Hergott, M. Kovacev, H. Merdji, C. Hubert, Y. Mairesse, E. Jean, P. Breger, P. Agostini, B. Carré, and P. Salières, Phys. Rev. A 66, 021801 (2002).

55. A. R. Libertun, X. Zhang, A. Paul, E. Gagnon, T. Popmintchev, S. Backus, M. M. Murnane, H. C. Kapteyn, and I. P. Christov, Appl. Phys. Lett. 84, 3903 (2004).

56. D. L. Ederer, Phys. Rev. Lett. 13, 760 (1964).

57. W. F. Chan, G. Cooper, X. Guo, G. R. Burton, and C. E. Brion, Phys. Rev. A 46, 149 (1992); see also erratum: Phys. Rev. A 48, 858 (1993).

58. U. Fano and J. W. Cooper, Rev. Mod. Phys. 40, 441 (1968).

59. U. Fano, Phys. Rev. 124, 1866 (1961).

60. O.-P. Sairanen, A. Kivimäki, E. Nõmmiste, H. Aksela, and S. Aksela, Phys. Rev. A 54, 2834 (1996).

61. D. L. Ederer and M. Manalis, J. Opt. Soc. Am. 65, 634 (1975).

62. D. L. Ederer, Appl. Opt. 8, 2315 (1969).

63. D. A Shaw, D. Cvejanović, G. C. King, and F. H. Read, J. Phys. B. 17, 1173 (1984).

64. J. Johnson, J. N. Cutler, G. M. Bancroft, Y. F. Hu, and K. H. Tan, J. Phys. B. 30, 4899 (1997).

65. A. Cummings and G. O’Sullivan, Phys. Rev. A 54, 323 (1996).

66. P. B. Corkum, Phys. Rev. Lett. 71, 1994 (1993).

67. G. Cerullo, M. Nisoli, S. Stagira, and S. De Silvestri, Opt. Lett. 23, 1283 (1998).

68. P. Baum, S. Lochbrunner, and E. Riedle, Opt. Lett. 29, 1686 (2004).

69. A. Giusti-Suzor, F. H. Mies, L. F. DiMauro, E. Charron, and B. Yang, J. Phys. B 28, 309 (1995). 
70. J. H. Posthumus, Rep. Prog. Phys. 67, 623 (2004).

71. K. Codling and L. J. Frasinski, J. Phys. B 26, 783 (1993). 
TABLE 1. Photon flux at the source for the most intense high-order harmonics shown in Figs. $6 a$ and $6 b$, obtained by integrating the number of counts on the CCD camera over a bandwidth of $2 \mathrm{eV}$ centered about the harmonic.

\begin{tabular}{ccccc}
\hline \hline Photon energy & HHG & Inlet pressure & HHG driver pulse & Output photons \\
$(\mathrm{eV})$ & medium & (Torr) & energy $(\mathrm{mJ})$ & per pulse \\
\hline 39.5 & $\mathrm{Ar}$ & 50 & 1.0 & $1 \times 10^{8}$ \\
43.6 & $\mathrm{Ar}$ & 50 & 1.4 & $2 \times 10^{8}$ \\
69.9 & $\mathrm{Ne}$ & 80 & 1.8 & $6 \times 10^{4}$ \\
\hline \hline
\end{tabular}




\section{FIGURE CAPTIONS}

FIG. 1 (COLOR ONLINE). Overview of the experimental apparatus, which consists of an amplified Ti:sapphire laser system, a capillary waveguide in which high-order harmonics are produced, and a vacuum system that houses a toroidal mirror, a sample gas cell in the interaction region, and a home-built soft x-ray spectrometer. BS: beamsplitter, FL: focal length, $\lambda / 2$ : zero-order half-waveplate, GV: gate valve, TMP: turbomolecular pump. The two arrows pointing toward the HHG capillary denote the positions of the gas inlets, whereas the remaining arrow pointing away from the HHG capillary denotes the connection to a scroll pump that is used to evacuate the front end of the capillary. The inset shows the layout for the soft x-ray optics. The first toroidal mirror (TM1) and second toroidal mirror (TM2, located in the soft $\mathrm{x}$-ray spectrometer) are used to refocus the divergent high-order harmonics into the sample gas cell and onto the detector plane, respectively. Spectral dispersion onto the CCD camera is achieved by means of a uniform line-spaced reflection grating $(\mathrm{G})$.

FIG. 2 (COLOR ONLINE). Close-up view of the optical arrangement used to achieve spatial overlap between the optical pump and soft x-ray probe beams at the interaction region with a $2^{\circ}$ crossing angle. The pick-off mirror consists of a dielectric-coated mirror with a $1 \mathrm{~mm}$ diameter hole drilled through the optic to allow passage of the soft x-ray probe beam, while the optical pump beam is reflected by the front surface. The interaction region is defined by the position of the sample gas cell. A collinear pump- 
probe overlap geometry can also be implemented with the pick-off mirror, at the expense of decreasing the available pump pulse energy.

FIG. 3. Schematic illustration of the sample gas cell. The gas cell consists of a PTFE cylindrical top and a stainless steel base. The PTFE top has $150 \mu \mathrm{m}$ or $200 \mu \mathrm{m}$ diameter holes drilled through it along the beam propagation axis to allow the pump and probe beams to pass through the cell. A Swagelok tube fitting welded to the stainless steel base enables the gas cell to be connected to the external sample gas manifold via a fluid feedthrough.

FIG. 4. Foci positions for various photon energies after spectral dispersion by the grating. The coordinates are referenced to the point of incidence on the grating by the soft x-ray beam. The line drawn through the points corresponds to the position of the CCD array plane. The inset shows the deviation of the foci position from the detector plane as a function of photon energy.

FIG. 5. Typical calibration curve for the home-built soft x-ray spectrometer, along with the marked positions of the $\mathrm{Al} L_{3}$-edge cut-off in the first four diffraction orders (O) and the $\mathrm{Xe}^{+2} P_{3 / 2} \rightarrow{ }^{2} D_{5 / 2}$ transition in the first two diffraction orders ( $\left.\mathbf{\square}\right)$.

FIG. 6. (a) High-order harmonic spectra obtained with 50 Torr of Ar as the HHG medium. The spectrum in the solid line is obtained with an input pulse energy of $1.0 \mathrm{~mJ}$ and exhibits relatively narrow individual peaks, whereas the spectrum in the dashed line 
is obtained with an input pulse energy of $1.4 \mathrm{~mJ}$ and shows broadband high-order harmonics. (b) High-order harmonic spectra obtained with 80 Torr of Ne as the HHG medium and an input pulse energy of $1.8 \mathrm{~mJ}$. The $\mathrm{Al} L_{3}$-edge cut-off at $72.64 \mathrm{eV}$ is apparent and substantiates the presence of a continuum underlying the discrete harmonic peaks.

FIG. 7. Spectra of the NIR driving pulse after propagation through the capillary waveguide. The spectrum in grey is obtained with an evacuated capillary. The dashed line corresponds to the spectrum taken with an Ar inlet pressure of 50 Torr and an input energy of $1.0 \mathrm{~mJ}$, whereas the solid black line is obtained with the same Ar inlet pressure, but with a higher input pulse energy of $1.4 \mathrm{~mJ}$. The former yields narrow individual peaks in the high-order harmonic spectrum, whereas the latter leads to broadband harmonic spectra.

FIG. 8. (a) Wavelength-tuning of high-order harmonics by increasing the Ne inlet pressure. An increase in inlet pressure leads to an observed linear increase in photon energy at the peak of the individual harmonics, as would be expected from the $\delta E_{q} \propto N$ relation delineated by Eq. (3). (b) The sensitivity of blueshifting with respect to inlet pressure shows a linear increase with harmonic order, i.e., $\delta E_{q} / N \propto q$. This trend verifies that blueshifting of the fundamental light in the capillary waveguide is the dominant mechanism that enables wavelength-tuning of the high-order harmonics. 
FIG. 9. Knife-edge scan traces for (a) the 25th harmonic produced with 50 Torr of Ar and $1.4 \mathrm{~mJ}$ of input pulse energy and (b) the 45th harmonic produced with 60 Torr of $\mathrm{Ne}$ and $1.8 \mathrm{~mJ}$ of input pulse energy. The measured beam waists are $24 \pm 1 \mu \mathrm{m}$ and $20 \pm 1$ $\mu \mathrm{m}$ for the 25 th and 45 th harmonic, respectively.

FIG. 10 (COLOR ONLINE). Wavelength-integrated spatial beam profiles of the highorder harmonics produced in (a) Ar and (b) Ne, recorded by a soft x-ray CCD camera positioned $30 \mathrm{~cm}$ after the sample focus. The area of the image is $2.0 \mathrm{~mm} \times 2.0 \mathrm{~mm}$. Intensity variations along the horizontal and vertical sections are shown below and to the left of each image, respectively.

FIG. 11. (a) High-order harmonic spectrum taken with an evacuated gas cell (dashed line) and with the gas cell filled with $51 \pm 1$ Torr of Xe (solid line). (b) Nonresonant absorption spectrum of Xe obtained from calculating the transmission at the peak of each high-order harmonic. Absorption cross-sections obtained from ref. [57] are also shown for comparison. (c) Resonant absorption lineshape due to the $\mathrm{Xe}{ }^{1} S_{0} \rightarrow$ $4 d^{-1}\left({ }^{2} D_{5 / 2}\right) 6 p\left({ }^{2} P_{3 / 2}\right)$ transition. (d) Resonant absorption lineshape due to the $\mathrm{Xe}{ }^{1} S_{0} \rightarrow$ $4 d^{-1}\left({ }^{2} D_{3 / 2}\right) 7 p\left({ }^{2} P_{1 / 2}\right)$ transition.

FIG. 12. (a) High-order harmonic spectra taken with an evacuated gas cell (dashed line) and with the gas cell filled with $17 \pm 1$ Torr of $\mathrm{CH}_{2} \mathrm{Br}_{2}$ (solid line). Note that use of a logarithmic scale. (b) Resonant absorption spectrum of $\mathrm{CH}_{2} \mathrm{Br}_{2}$ showing two broad transitions separated by the $\mathrm{Br} 3 d$ spin-orbit splitting of $1 \mathrm{eV}$. 
FIG. 13. Time-evolution of the $57.0 \mathrm{eV}$ harmonic from $-80 \mathrm{fs}$ to $+80 \mathrm{fs}$, in steps of $10 \mathrm{fs}$. The circle on each spectrum is located at $57.0 \mathrm{eV}$. The decrease in spectral intensity on going from 0 fs to $+10 \mathrm{fs}$ is indicative of the onset of transient absorption due to formation of $\mathrm{Xe}^{2+}$ by sequential strong-field ionization of $\mathrm{Xe}$ atoms with an $800 \mathrm{~nm}$ pump pulse.

FIG. 14. Transient absorption spectrum showing both $\mathrm{Xe}^{+}$and $\mathrm{Xe}^{2+}$ produced by optical strong-field ionization of Xe atoms. The peak intensity of the pump pulse is $6.2 \times 10^{14}$ $\mathrm{W} / \mathrm{cm}^{2}$. The $\mathrm{Xe}^{+}$absorption features observed at $55.4 \mathrm{eV}\left({ }^{2} P_{3 / 2} \rightarrow{ }^{2} D_{5 / 2}\right)$ and $56.1 \mathrm{eV}$ $\left({ }^{2} P_{1 / 2} \rightarrow{ }^{2} D_{3 / 2}\right)$ are collected by employing the weak continuum present in the high-order harmonic spectrum. The $\mathrm{Xe}^{2+}$ absorption at $57.0 \mathrm{eV}$ consists of two unresolved transitions at $56.94 \mathrm{eV}\left({ }^{3} P_{2} \rightarrow{ }^{1} D_{2}\right)$ and $57.11 \mathrm{eV}\left({ }^{3} P_{2} \rightarrow{ }^{3} D_{3}\right)$.

FIG. 15. Time trace showing the transient absorption at $57.0 \mathrm{eV}$ as a function of pumpprobe time delay. The data is fit to a convolution of a step function with a Gaussian of 13 \pm 3 fs FWHM. This value sets the upper bound for the duration of the soft x-ray pulse. 
FIGURE 1 (COLOR ONLINE)

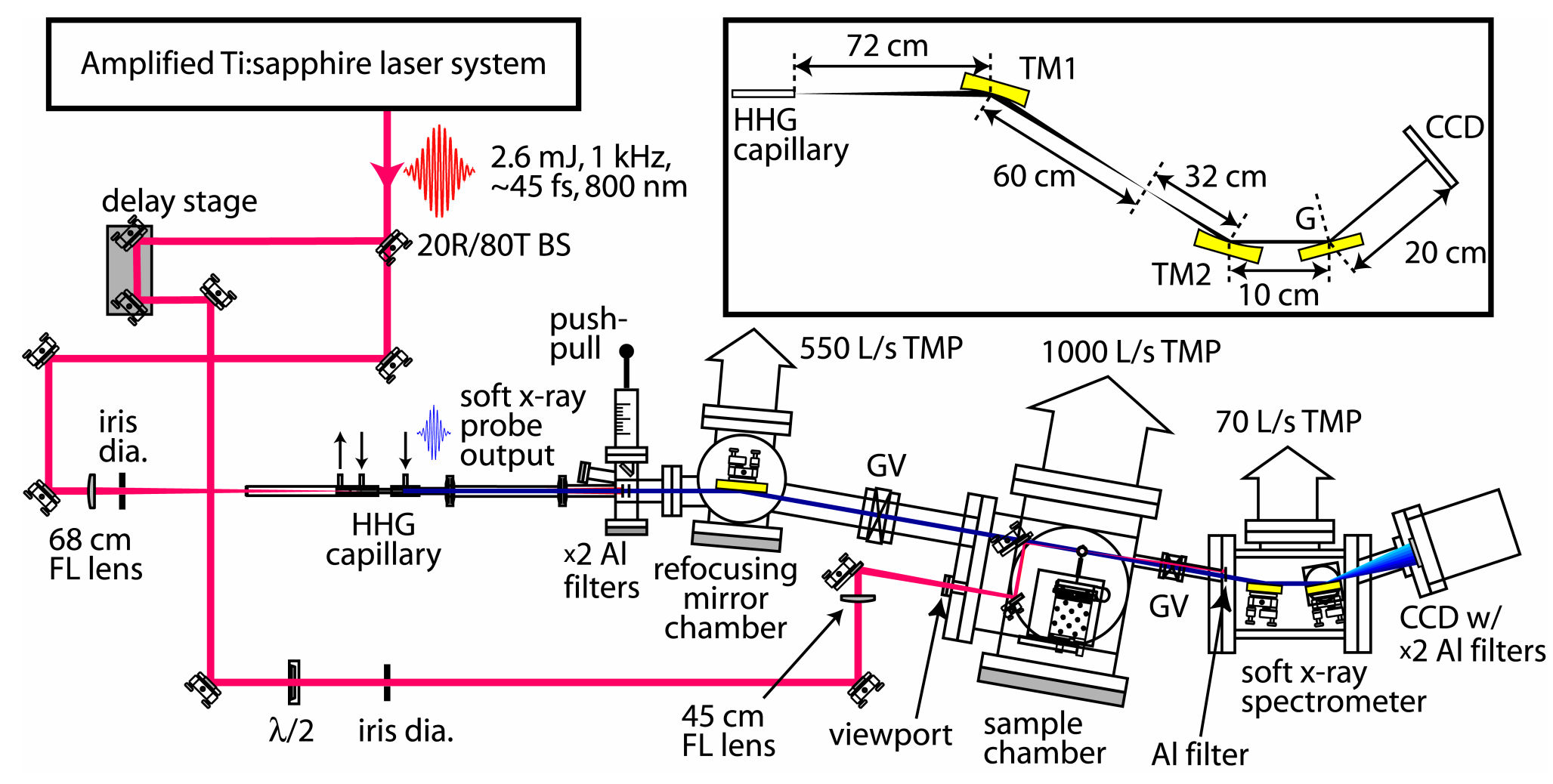


FIGURE 2 (COLOR ONLINE)

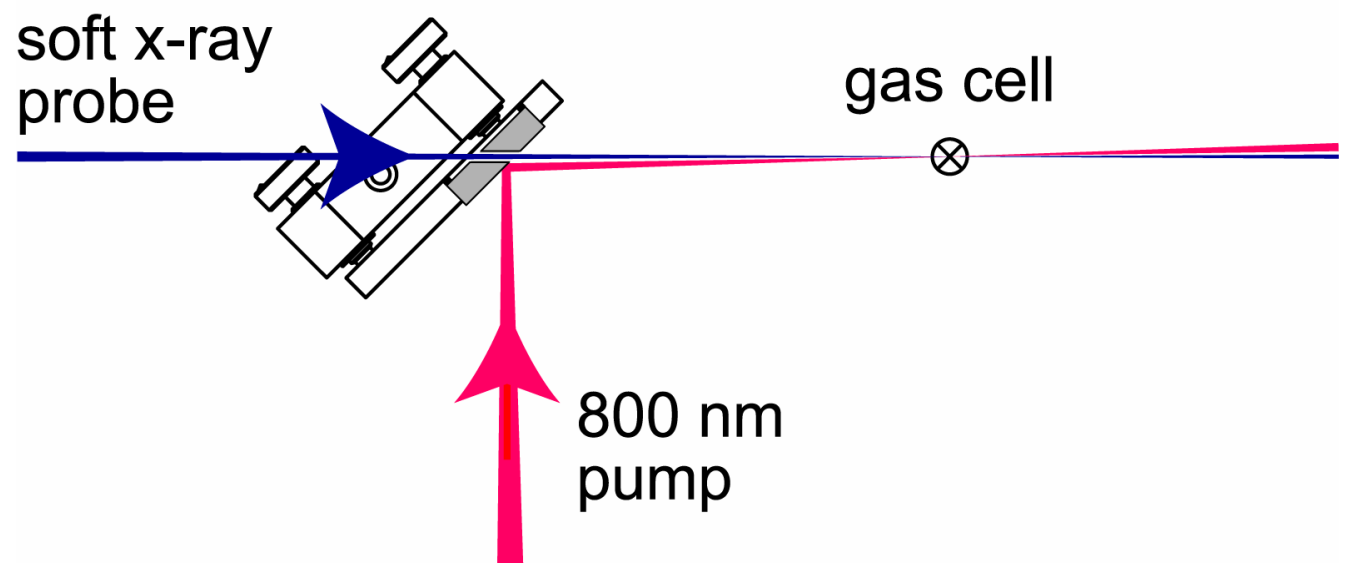


FIGURE 3

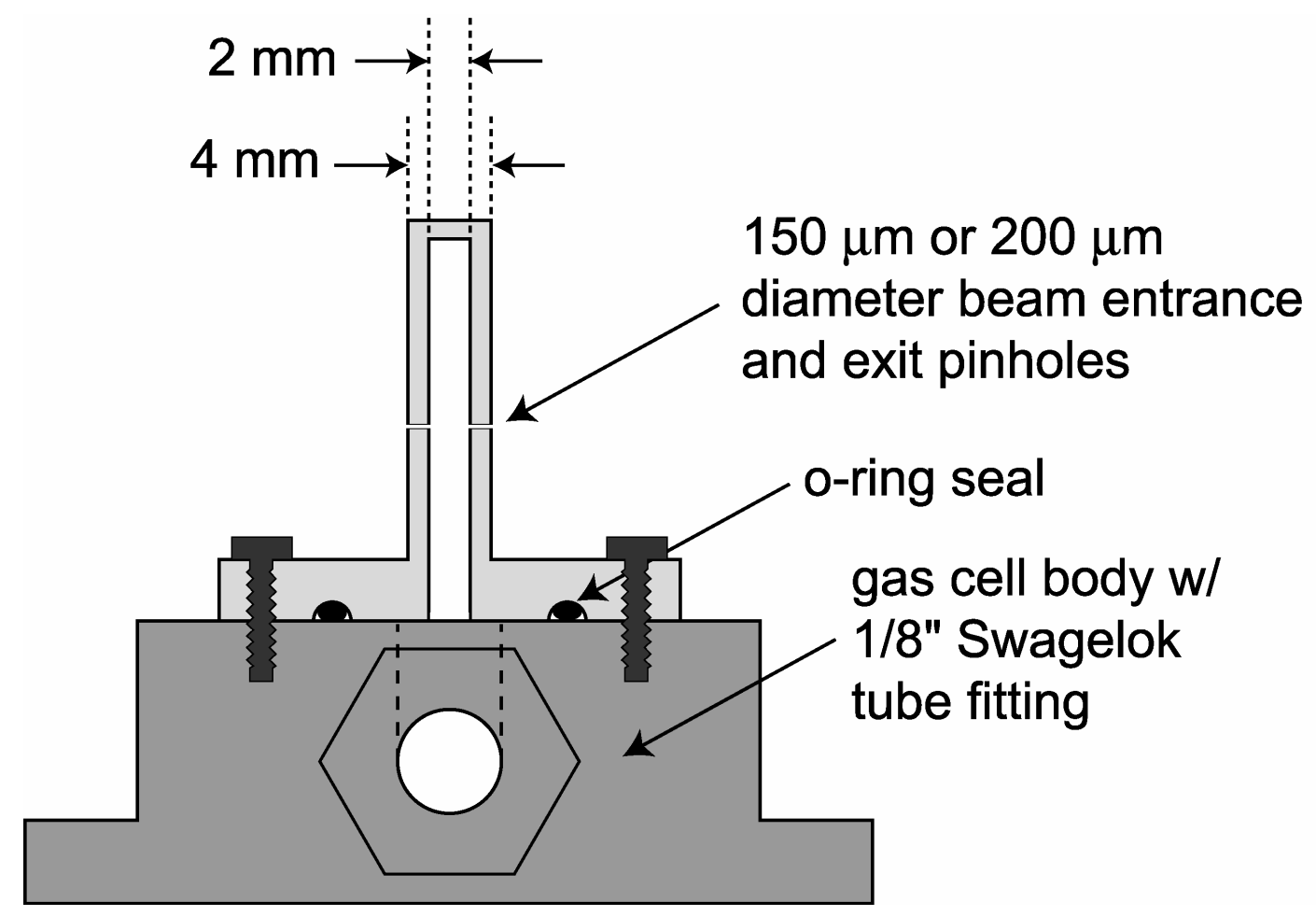




\section{FIGURE 4}

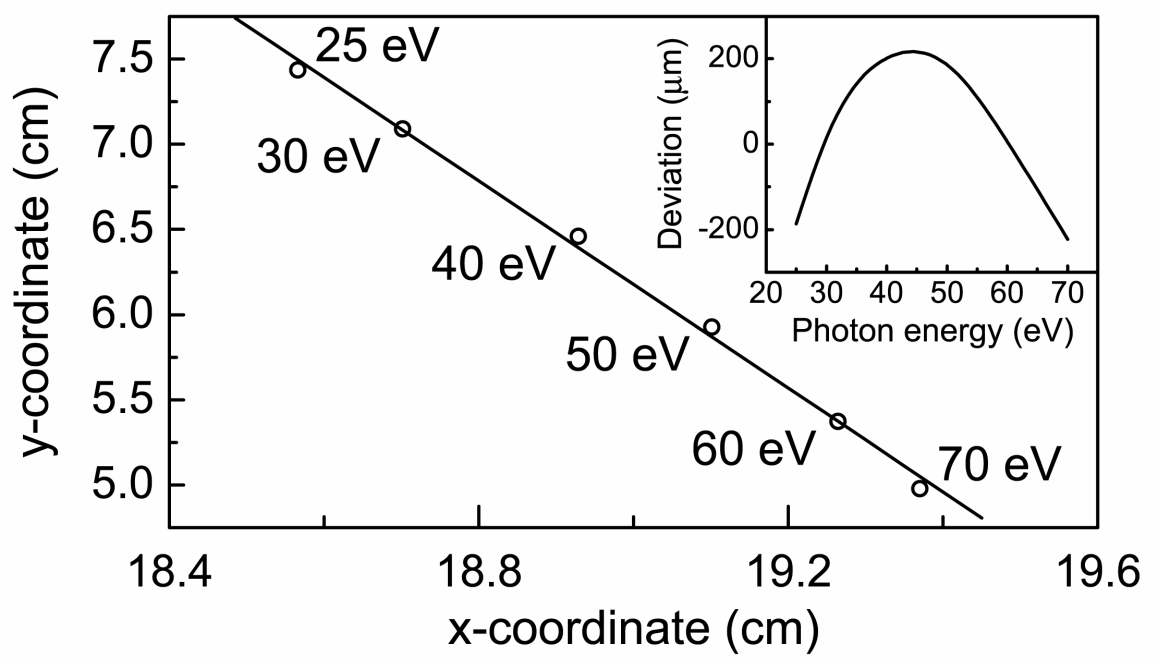




\section{FIGURE 5}

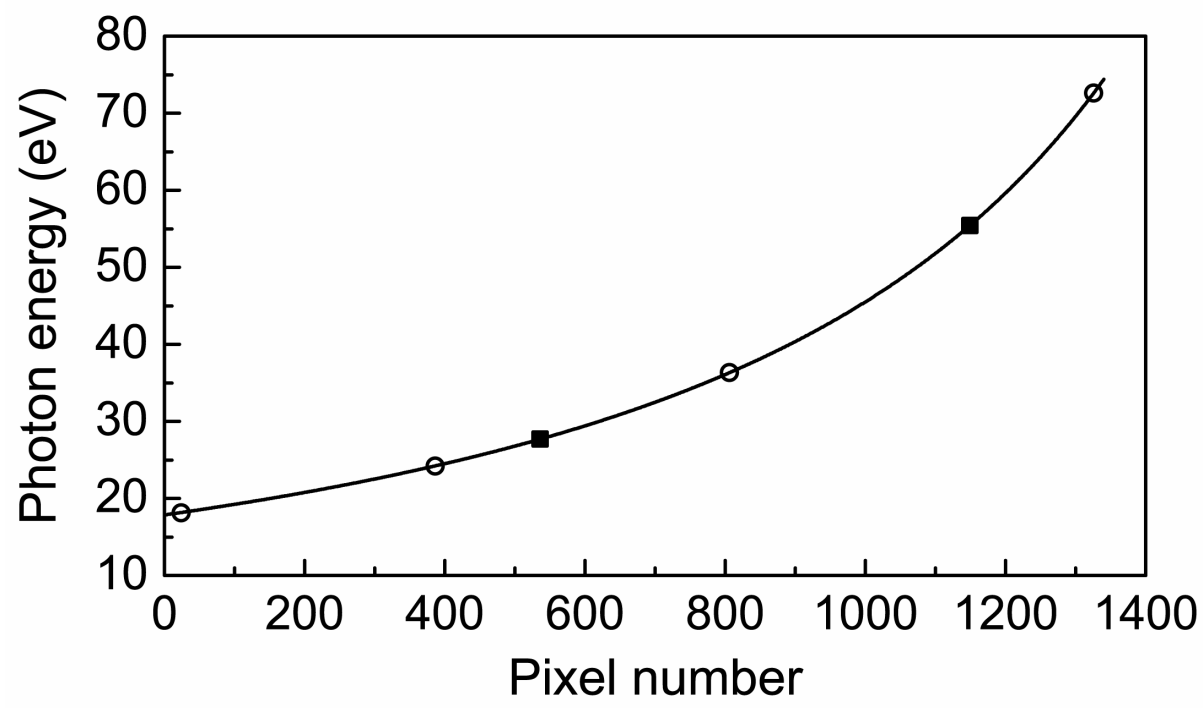


FIGURE 6

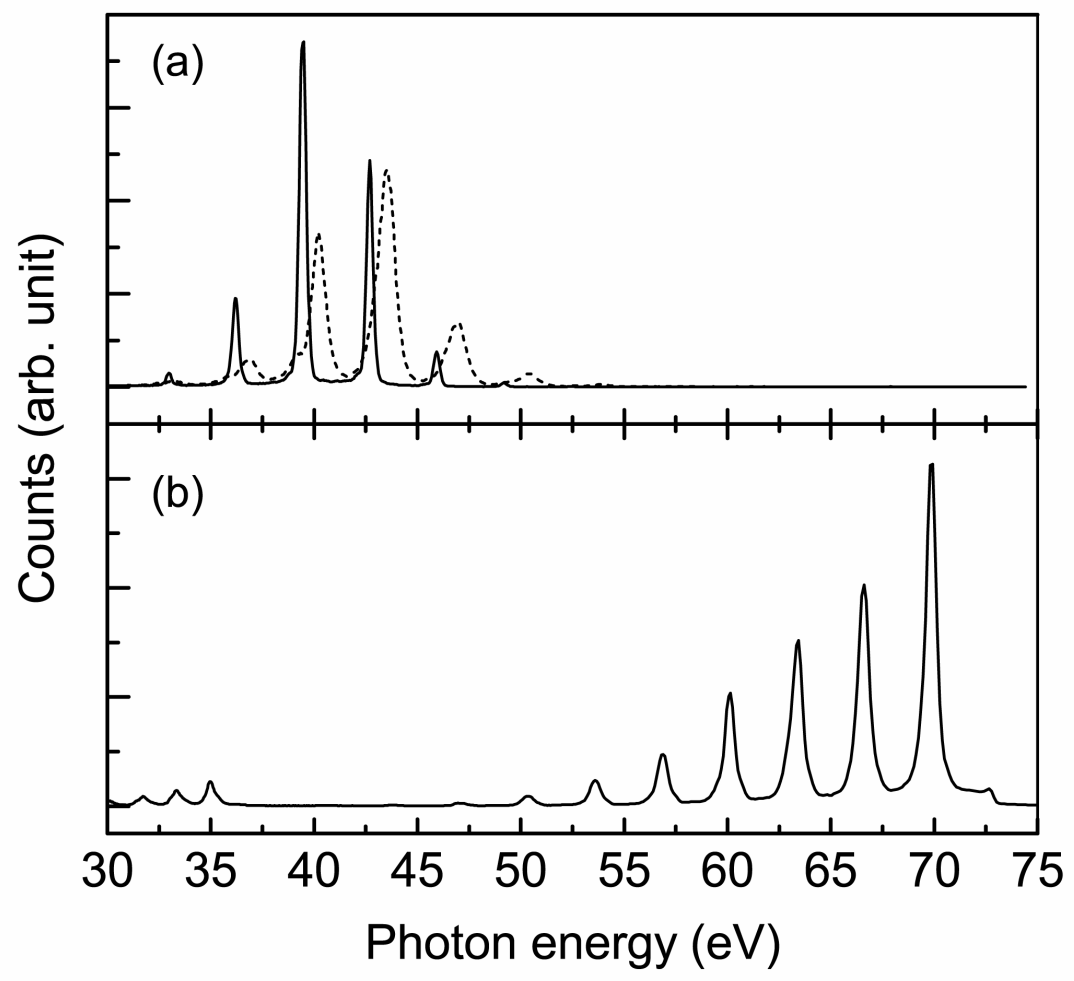




\section{FIGURE 7}

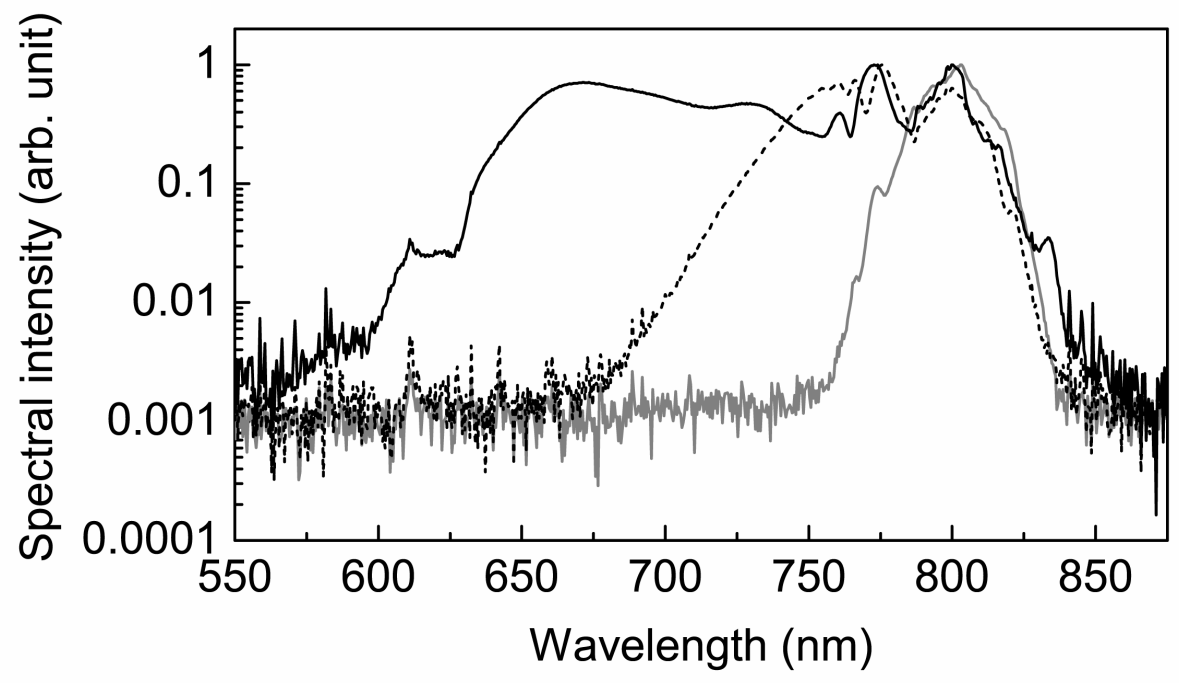


FIGURE 8
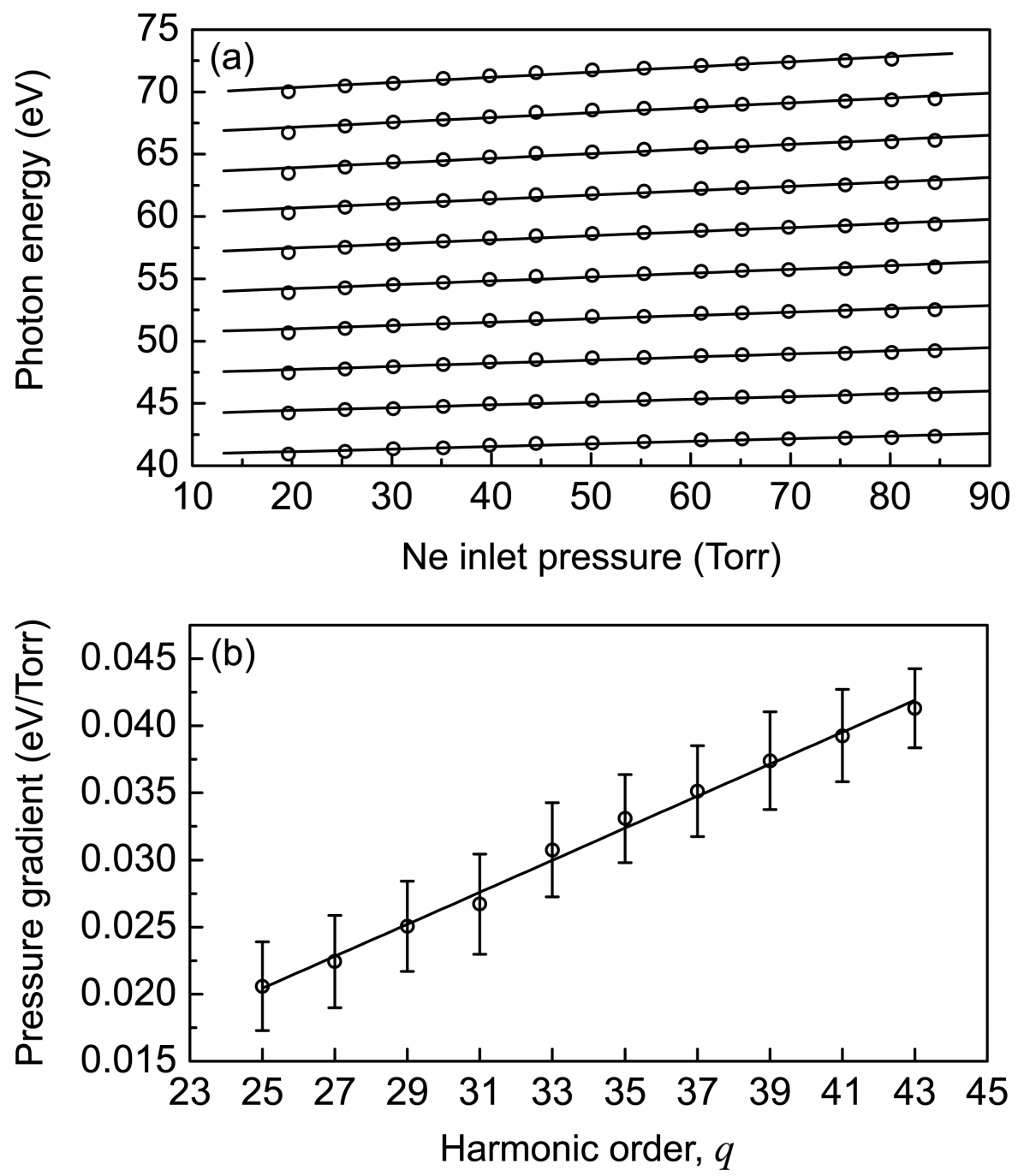
FIGURE 9
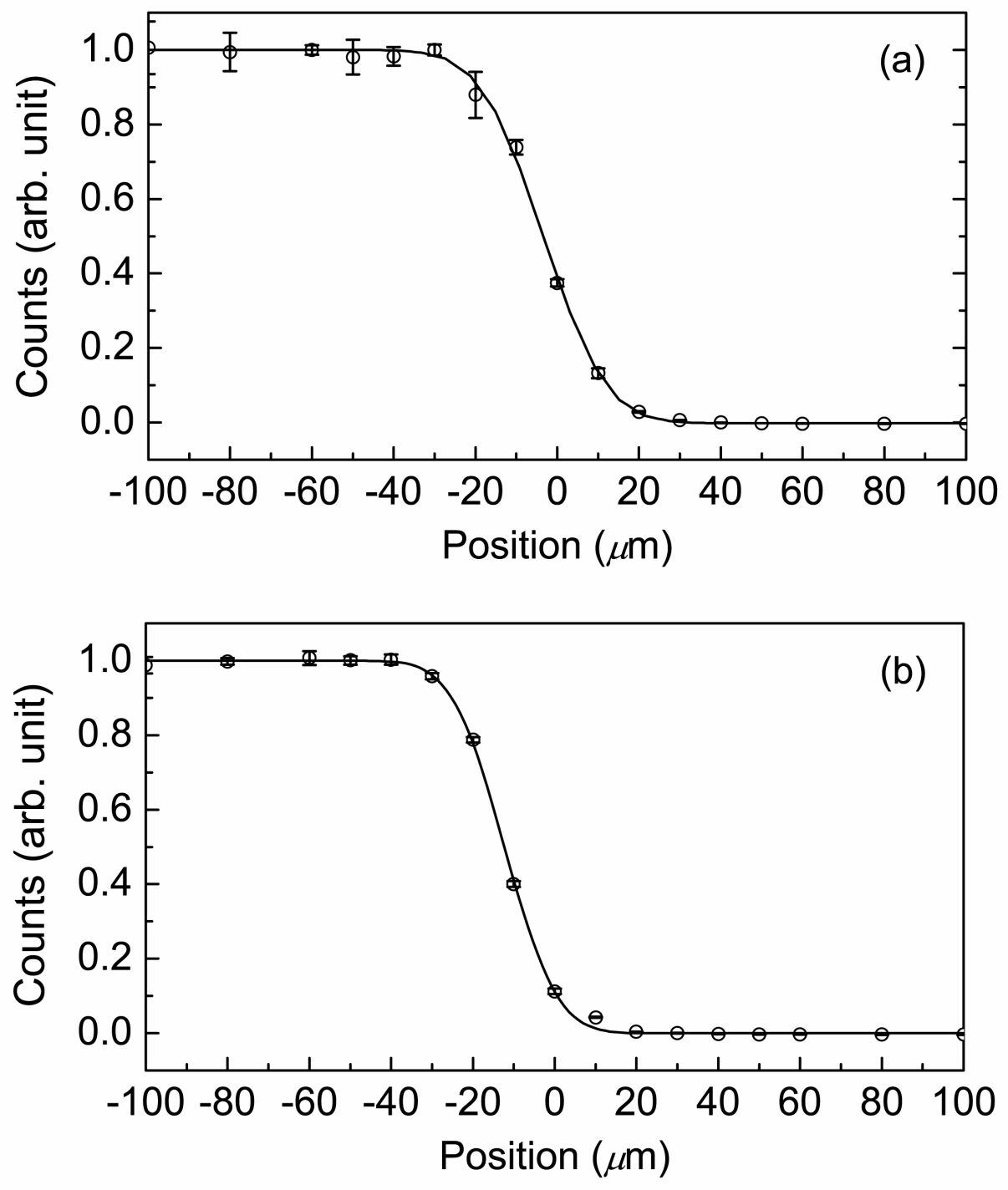
FIGURE 10 (COLOR ONLINE)
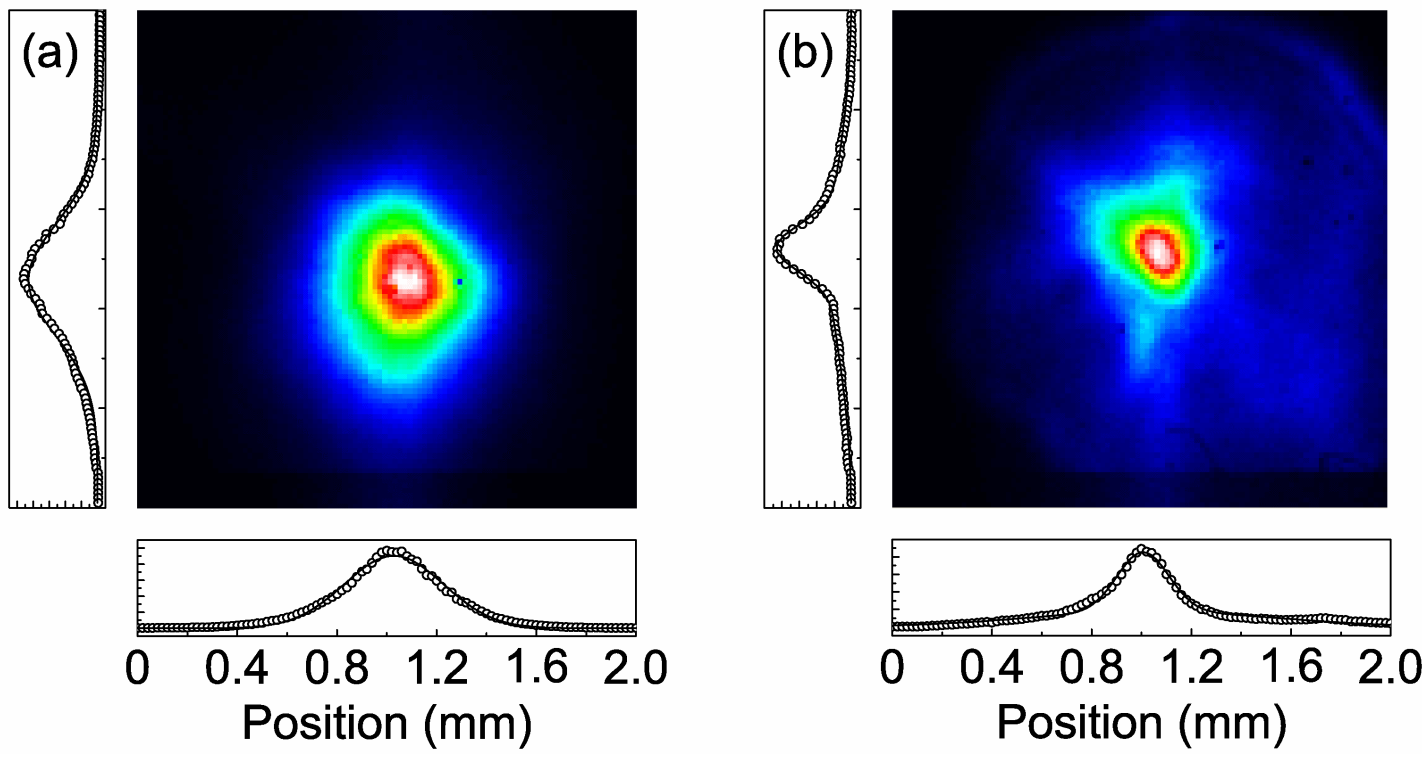
FIGURE 11
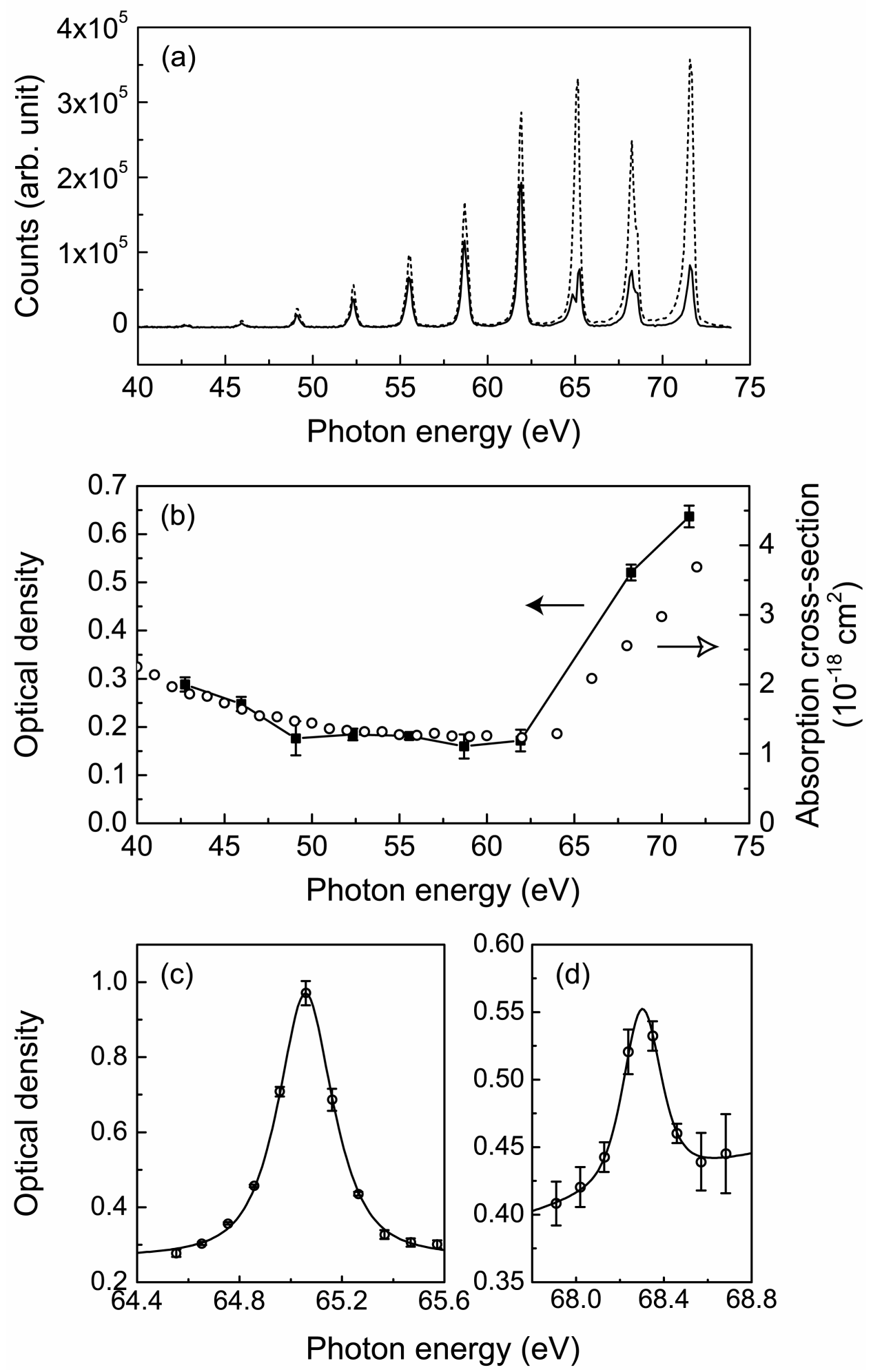
FIGURE 12
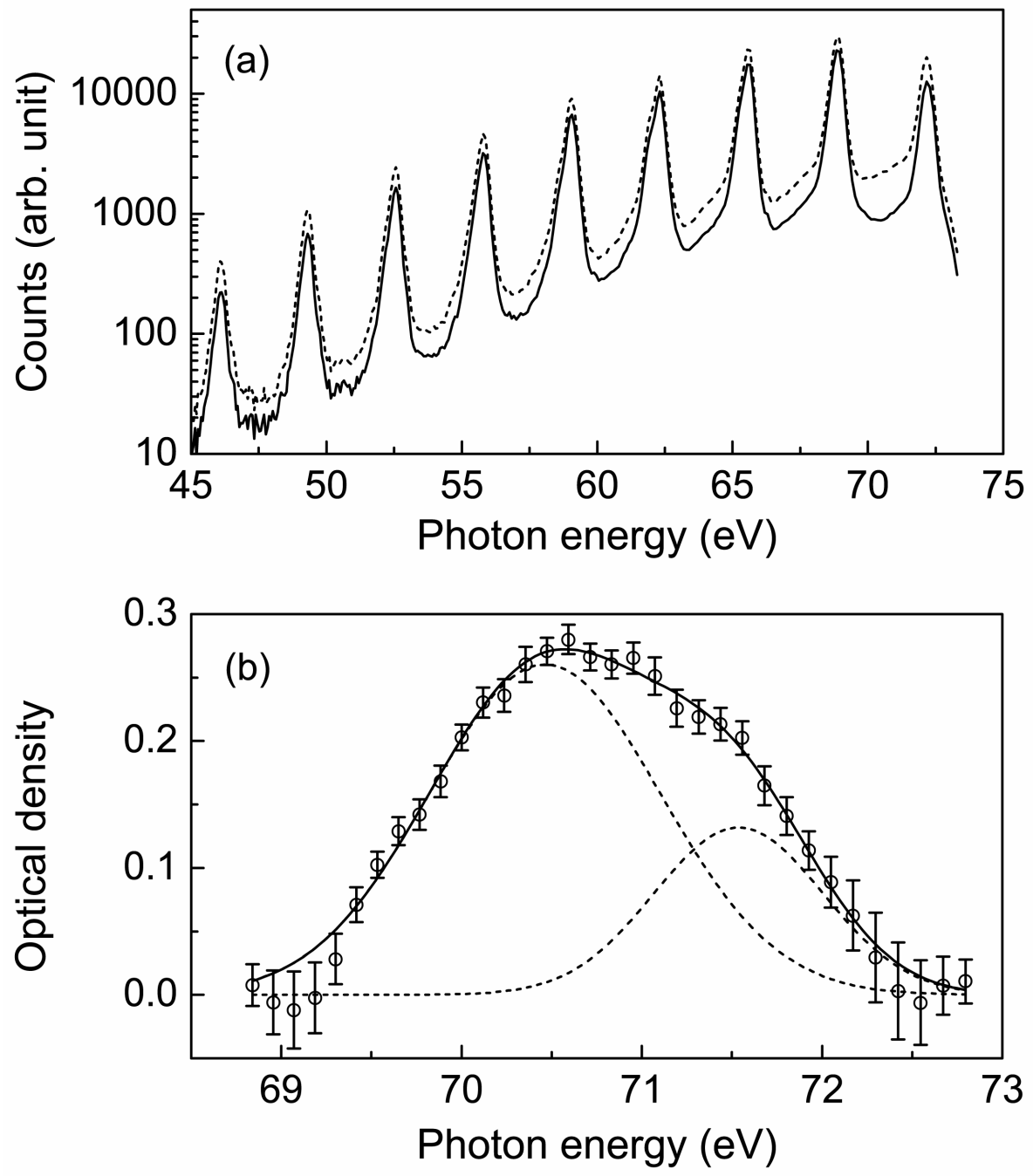
FIGURE 13

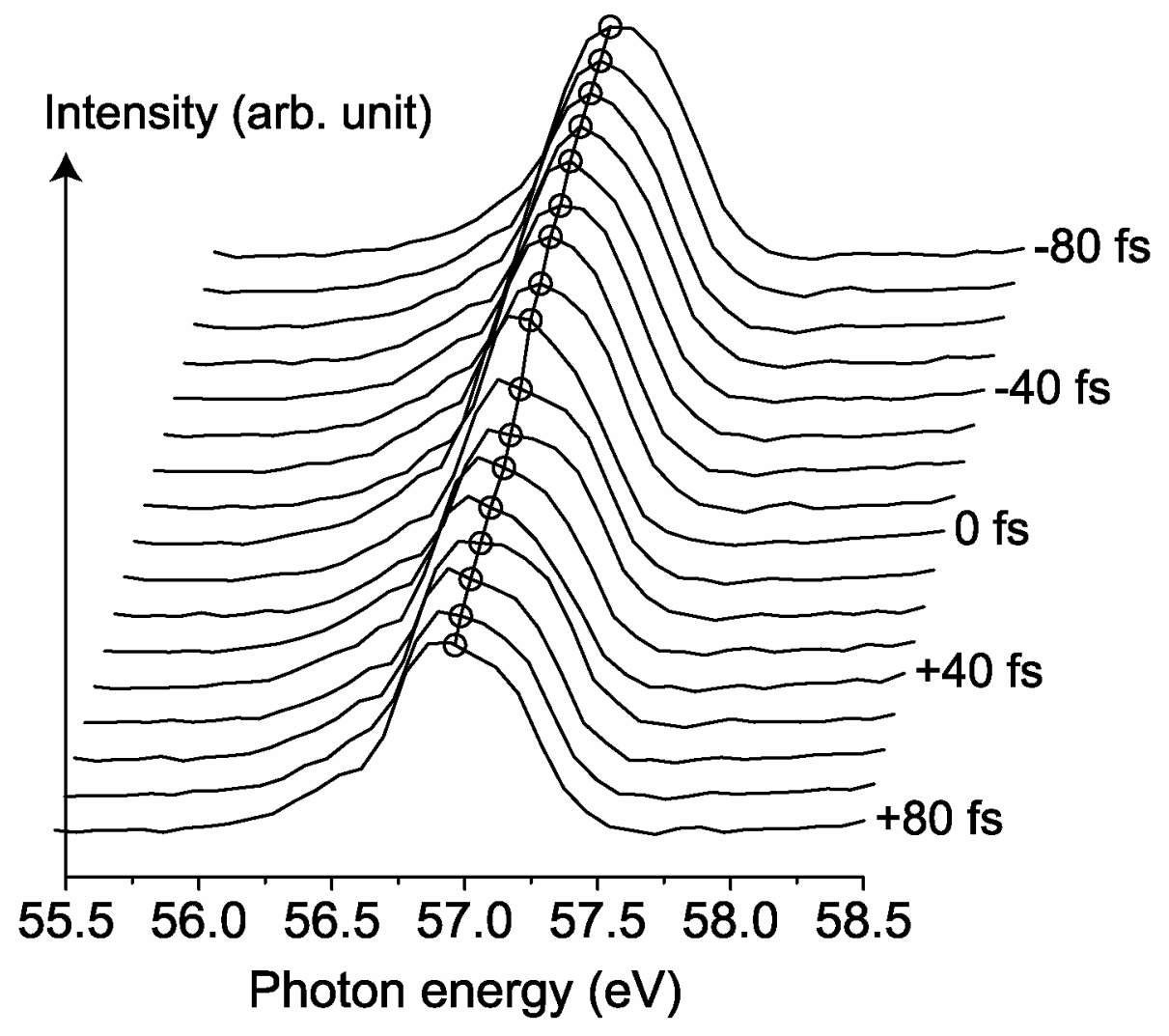




\section{FIGURE 14}

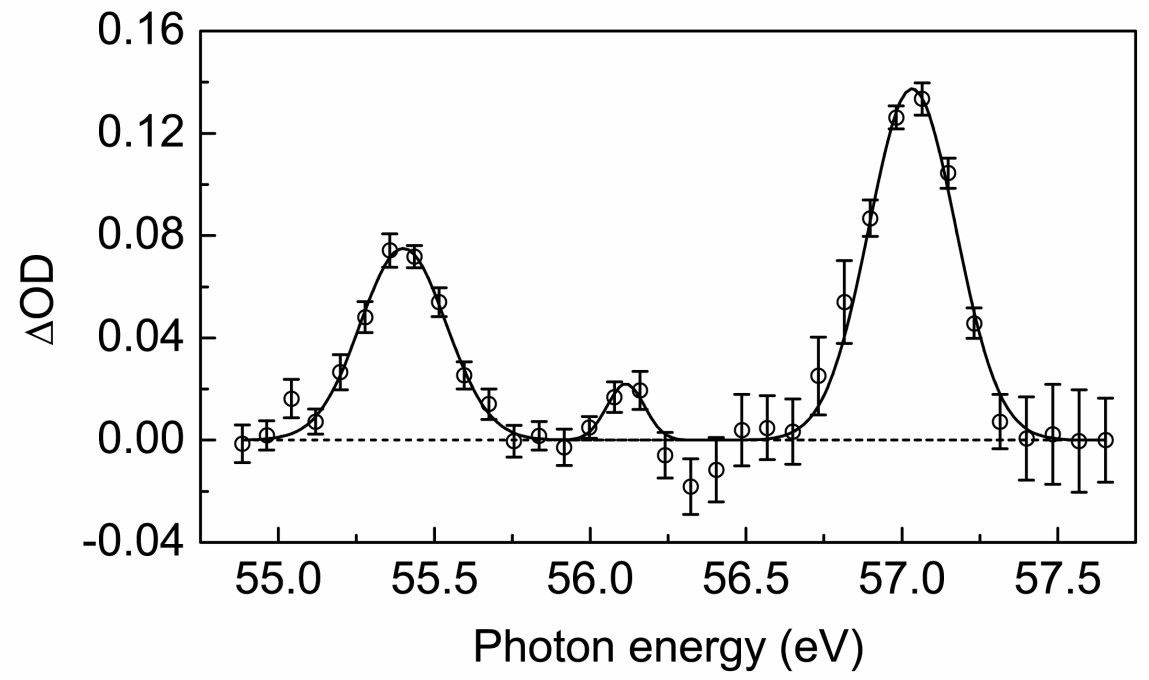




\section{FIGURE 15}

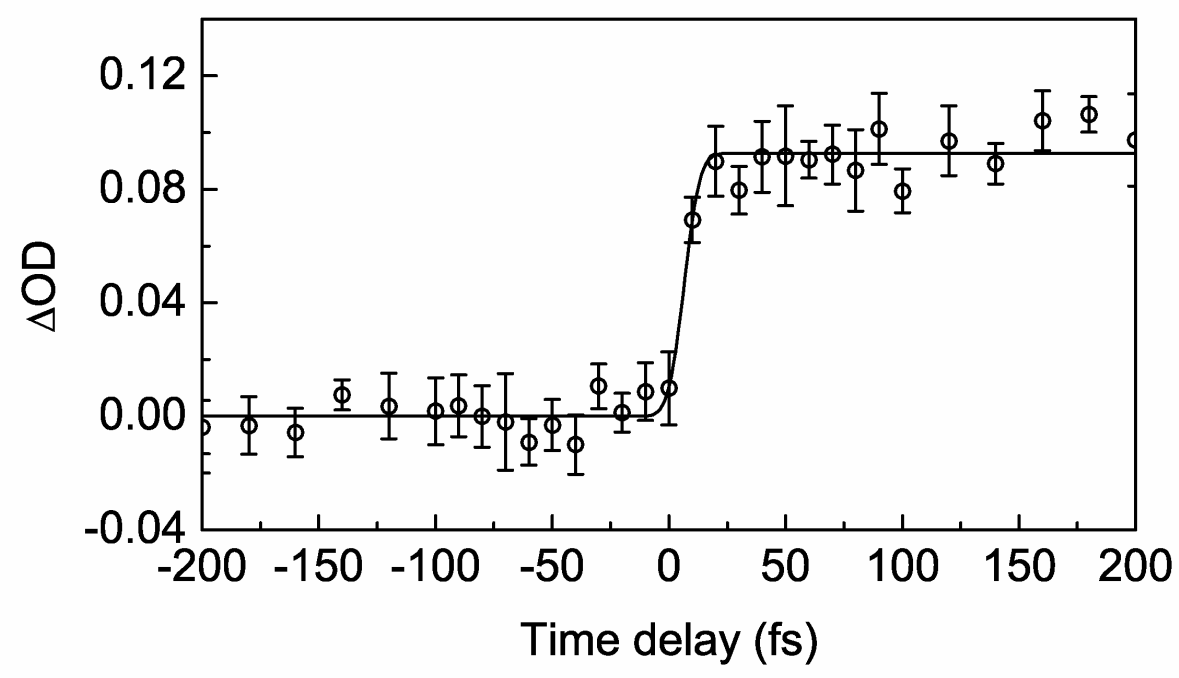

